\title{
Engineered Bacteriophage T4 Nanoparticle as a Potential Targeted Activator of HIV-1 Latency in CD4+ Human T-cells
}

Himanshu Batra ${ }^{1}$, Jingen Zhu ${ }^{1}$, Swati Jain ${ }^{1}$, Neeti Ananthaswamy ${ }^{1}$, Marthandan Mahalingam ${ }^{1}$, Pan Tao ${ }^{1}$, Camille Lange ${ }^{2}$, Chaojie Zhong ${ }^{3}$, Mary F. Kearney ${ }^{2}, \mathrm{Haitao}^{\mathrm{Hu}^{3 *}}$, Frank Maldarelli ${ }^{2^{*}}$, Venigalla B. Rao ${ }^{1^{*}}$

${ }^{1}$ Bacteriophage Medical Research Center, Department of Biology, The Catholic University of America, Washington, D.C, United States of America

${ }^{2}$ National Cancer Institute, National Institutes of Health, Frederick, MD, United States of America

${ }^{3}$ Department of Microbiology and Immunology, University of Texas Medical Branch, TX, United States of America

*Correspondence: $\underline{\text { haihu@utmb.edu; fmalli@mail.nih.gov; rao@cua.edu }}$ 


\begin{abstract}
The latent HIV-1 reservoir containing stably integrated and transcriptionally silent proviruses in CD4+ $\mathrm{T}$ cells is a major barrier for virus eradication. Targeted reactivation of the latent reservoir remains a major challenge in establishing a path for an HIV-1 cure. Here, we investigated the possibility of reactivating the HIV-1 reservoir by targeting engineered bacteriophage T4 capsid nanoparticles to reservoir cells. The surface lattice of the $120 \times 86 \mathrm{~nm}$ phage capsid was arrayed with CD4 binding ligands such as recombinant CD4DARPin or the HIV-1 gp140 envelope protein. When exposed to either PBMCs or the resting CD4+ T cells in vitro, these nanoparticles caused $\mathrm{T}$ cells activation without inducing global $\mathrm{T}$ cell activation. Furthermore, the nanoparticles reactivated HIV-1 proviral transcription that led to virus assembly and release in the J-Lat cells, a cell line model of HIV-1 latency. Intriguingly, the observed T cell activation and HIV-1 latency reversal did not occur through the classic PKC or NFAT pathways suggesting the involvement of a yet unknown pathway. These studies demonstrate that engineered non-infectious bacteriophages could be potentially exploited for HIV-1 cure and other targeted T cell therapies.
\end{abstract}




\section{INTRODUCTION}

Bacteriophages (phages) are present in diverse ecosystems ranging from the ocean to the human body (Salmond and Fineran, 2015). As the most abundant foreign organism in the human body, phages co-exist with human cells like other microflora such as bacteria and fungi (Gensollen et al., 2016). They home in a broad spectrum of organs, though prominently reside in the gut's distal region, either as prophage in a bacterial host or free virion embedded in the mucus layer (Barr et al., 2013). Some phages in the gut potentially translocate through the epithelial monolayer into lamina propria and submucosal layer, where they can interact with the immune system (Nguyen et al., 2017). While phages do not directly infect the human host, they are shown to potentially stimulate the immune system (Duerkop and Hooper, 2013).

Phage T4 decorated with pathogen epitopes mimic PAMPs (pathogen-associated molecular patterns) of natural viruses and can stimulate strong innate as well as adaptive immune responses (Tao et al., 2019). Induction of innate immune responses plays a critical role in HIV-1 infection and can reverse HIV-1 latency. Toll-Like Receptors (TLR) agonists such as TLR7, TLR9, TLR8, and TLR5 are shown to reactivate latent HIV-1 and are being used in the clinical trials for HIV-1 cure (Tsai et al., 2017).

During HIV-1 infection, the virus enters the body and targets CD4+ T cells, and once inside the cell, the virus makes its way to the nucleus and inserts itself into the host cell's genomic DNA. Most of the infected CD4+ T-cells actively produce new viruses, but in a fraction of cells, the virus enters into a latent phase where it integrates into the human genome and remains transcriptionally silent. A collection of such cells carrying intact HIV-1 "proviruses" constitute the latent "reservoir" (Churchill et al., 2016; Maldarelli et al., 2014). Although combinational antiretroviral therapy 
(cART) effectively controls the infection, it is not curative as it does not target the integrated proviruses. Hence, viral rebound occurs by reactivation of a small pool of proviruses when treatment is discontinued (Chun et al., 1999). Thus, reducing or eliminating the latent reservoir is essential to achieve "remission" or "cure." One of the potential curative strategies is "shock and kill" which involves reversing the latent state by inducing HIV-1 transcription and subsequently killing the infected cells by the immune system.

Here, we describe the potential use of CD4 receptor-targeting T4 nanoparticles (CD4DARP-T4-NPs) for activation of CD4+ T cells. Phage T4 is a myovirus that infects the $E$. coli bacterium and belongs to the Order Caudovirales, representing the most abundant phage order in the intestine. Phage T4 has a large $120 \times 86 \mathrm{~nm}$ prolate head and a $140 \mathrm{~nm}$-long contractile tail to which six long tail fibers are attached through a baseplate. The elongated T4 icosahedron is built with 930 molecules of the major capsid protein gp23* ("*" represents the cleaved mature form), 55 copies of gp24* at eleven of the twelve vertices, and 12 copies of the portal protein gp20 at the unique twelfth vertex (Chen et al., 2017). The portal vertex is a ring structure with a central channel having a diameter of 3.5-4 nm through which DNA enters the capsid during packaging and exits during infection (Sun et al., 2008). The surface of the T4 capsid is arrayed with two nonessential outer capsid proteins, Soc (small outer capsid protein) $(9.1 \mathrm{kDa} ; 870$ copies per capsid) and Hoc (highly antigenic outer capsid protein) (40.4 kDa; 155 copies per capsid) (Fokine et al., 2004; Qin et al., 2010; Yanagida et al., 1984). Soc is a tadpole-shaped molecule and binds at the quasi threefold axes as a trimer. Each soc subunit acts as a "molecular clamp" by clasping two adjacent capsomers and, therefore, provides stability at high alkaline $\mathrm{pH}(\mathrm{pH} 11)$ (Qin et al., 2010). Hoc, on the other hand, is a $170 \AA$-long fiber containing a string of four Ig-like domains with the Cterminal domain bound to the center of each gp23* capsomer. One hundred and fifty-five 
symmetrically positioned Hoc fibers emanate from the T4 head (Fokine et al., 2011). Inside the capsid is a 171-kb linear dsDNA genome packaged by an ATP-powered pentameric molecular motor (gp17). The T4 phage tail is connected to the head via portal and neck proteins (Fig S1 A).

Over 40-years of our genetic, biochemical, and structural analyses allowed us to manipulate the complex genetic program of phage T4 life cycle and establish an in vitro system to generate engineered T4 nanoparticles (Black and Rao, 2012; Rao and Black, 2010; Sun et al., 2008). Purified empty shells (head or capsid) lacking the outer capsid proteins Soc and Hoc, neck, and tail could be filled with any linear DNA using the powerful T4 DNA packaging motor (gp17 ATPase) and the surface could be arrayed with $\sim 1,025$ molecules of foreign proteins fused to Hoc and Soc. These nanoparticles carrying a payload of genes and proteins could then be delivered into human cells for various biomedical applications such as vaccine delivery and gene therapy.

Here, by arraying CD4-binding DARPin (Designed Ankyrin Repeat Proteins) on T4 capsid lattice, we prepared nanoparticles that can efficiently target these nanoparticles (CD4DARPin-T4-NPs) and the associated payloads to CD4+ T cells. Remarkably, we found that these nanoparticles nearly completely titrate the CD4+ T cells from human PBMCs and cause their activation. However, the activation is not global and does not lead to cytokine storm or involve the classic PKC or NFAT pathways. Furthermore, these nanoparticles cause reactivation of proviral genomes resident in HIV-1 latency model cell lines and lead to virion assembly and release. Our studies demonstrate the potential use of engineered T4-NPs as HIV-1 viral mimics to target and activate CD4+ T cells, which can be exploited as a more deliberate "shock and kill" strategy for HIV-1 cure. 


\section{RESULTS}

\section{Assembly of CD4DARPin-T4 Nanoparticles}

The shock-and-kill strategy for HIV cure suggests that reactivation of virus transcription in CD4+ T cells containing a stably integrated and transcriptionally silent form of HIV-1 provirus, is the first essential step to eliminate the latent reservoir in ART-treated HIV-infected individuals (Archin et al., 2014). In untreated individuals, HIV-1 activates CD4+ T cells through its envelope glycoprotein interaction with the CD4 receptor. In individuals with viremia suppressed on cART, neither low viral copies ( $\sim 50$ copies/ml or less) nor the soluble gp120 or gp140 shed from the viruses, are sufficient for proviral reactivation (Klasse and Moore, 2004). Therefore, we sought to develop T4 nanoparticles (NPs) that can target the CD4 receptor with a rationale that such particles might mimic HIV-1 interaction and activate CD4+ $\mathrm{T}$ cells (Fig. 1A). For this, we decided to decorate our T4-NPs with CD4 binding DARPin owing to its unique specificity and high affinity (1.5-83 nM) for the CD4 receptor. CD4DARPin has been previously selected by in vitro evolution using DARPin libraries (Schweizer et al., 2008). T4-NPs were assembled by sequential incorporation of purified biomaterials to generate HIV-1 structural mimics (Fig.1B). We hypothesized that the T4 phage capsid structure mimicking the molecular patterns associated with eukaryotic virus capsids and CD4 targeting might activate the latent HIV-1 proviruses (Fig. 1C). 
A
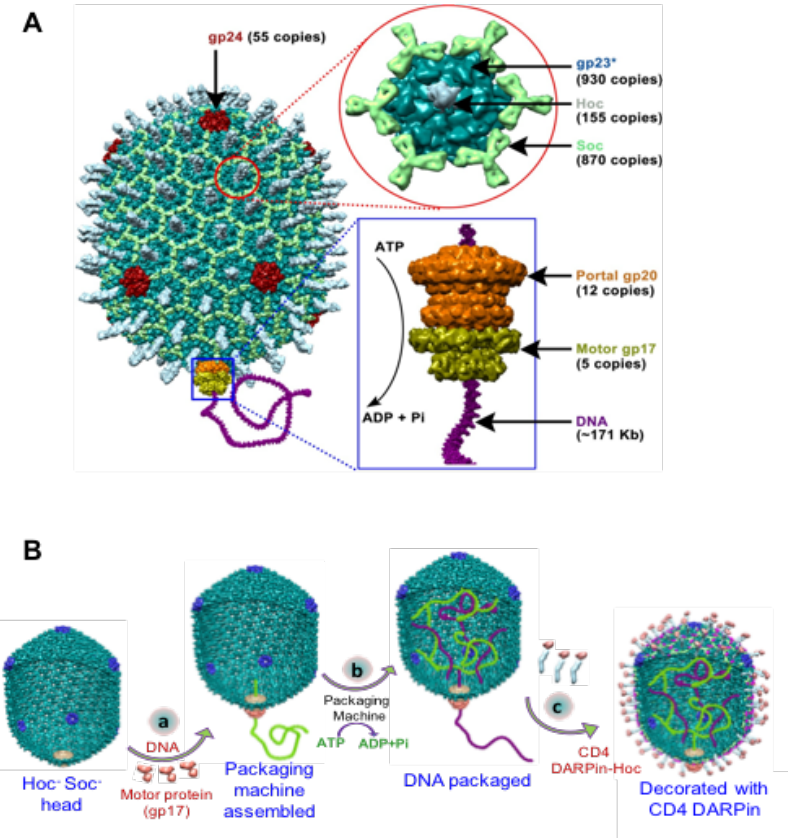

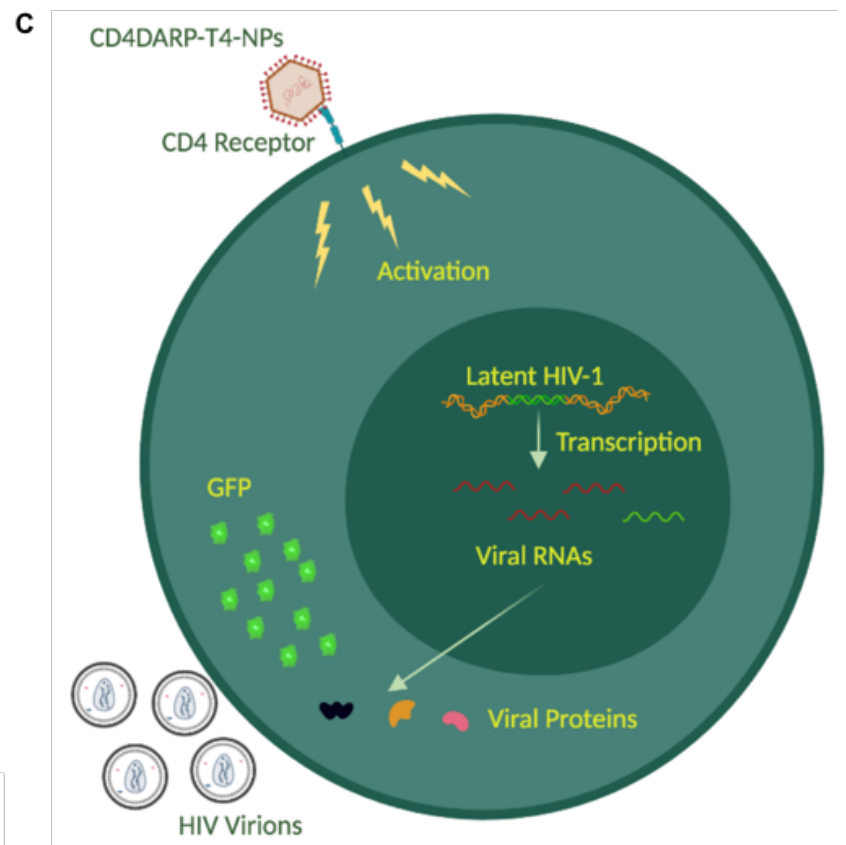

Fig 1. Assembly of CD4DARP-T4-NPs. A. Components of bacteriophage T4-NPs showing structural model of phage T4 head (capsid), pentameric gp24* vertices are shown in dark red, enlarged capsomer (hexamer) shows the arrangement of the major capsid protein gp23* (dark green), Soc trimers (light green), and Hoc fiber (cyan) and enlarged portal vertex showing gp20 dodecamer (brown) and pentameric DNA packaging motor gp17 (yellow). B. Preparation of phage T4-NPs. To prepare phage T4 -NPs, empty Hoc ${ }^{-} \mathrm{Soc}^{-}$phage T4 head (the cut-out of the head shows both the exterior and the interior) (a) were packaged with DNA molecules by gp17 motor by using the energy from ATP hydrolysis (b) CD4 targeting DARPin fused to Hoc were displayed on the heads (c). C. CD4 targeted phage T4-NPs mediate T cell activation and HIV-1 latency reversal. T4-NPs bind specifically to CD4 receptor, which leads to transcriptional activation of HIV-1 provirus and subsequent translation of viral mRNA to synthesize HIV proteins in addition to GFP which acts as a marker for activation. The virions assemble, bud and release from the activated cell.

The T4 -NPs were assembled starting from an empty capsid shell isolated from a neck- and tail-minus T4 phage mutant-infected E. coli cells (Fig. S1 B, C \& D). To confer CD4+ T cells targeting ability, we displayed high-affinity CD4 DARPin fused to N-terminus of Hoc (Fig. S2, A). The N-terminus of Hoc was used as it is projected away at $\sim 170 \AA$ distance from the capsid wall, whereas the C-terminal domain of the Hoc fiber having the capsid binding site would be closest to the capsid (Sathaliyawala et al., 2010). The CD4DARPin-Hoc fusion proteins; and CD4 DARPin, Hoc, and cell penetration peptide (CPP)-TAT-Hoc that were overexpressed and purified 
from E. coli were used as controls (Fig. S2, B). In addition, the fusion partners, CD4DARPin and Hoc, acted as positive and negative controls respectively. The cell penetration peptide CPP-TAT is a 14-aa peptide rich in basic amino acids that was shown to facilitate passage of attached cargo molecules across the cell membrane (Milletti, 2012). To test whether fusion of Hoc and CD4DARPin have affected the binding of CD4DARPin to CD4 receptor, an ELISA was performed using a soluble human CD4 receptor with full-length extracellular domain (amino acids 1-370). The data showed strong binding of CD4DARPin, CD4DARPin-Hoc fusion proteins, and JRFL-SOSIP-664 soluble trimers to soluble CD4 but not to the negative controls, Hoc and CPPTAT-T4 Hoc (Fig. S2, C).
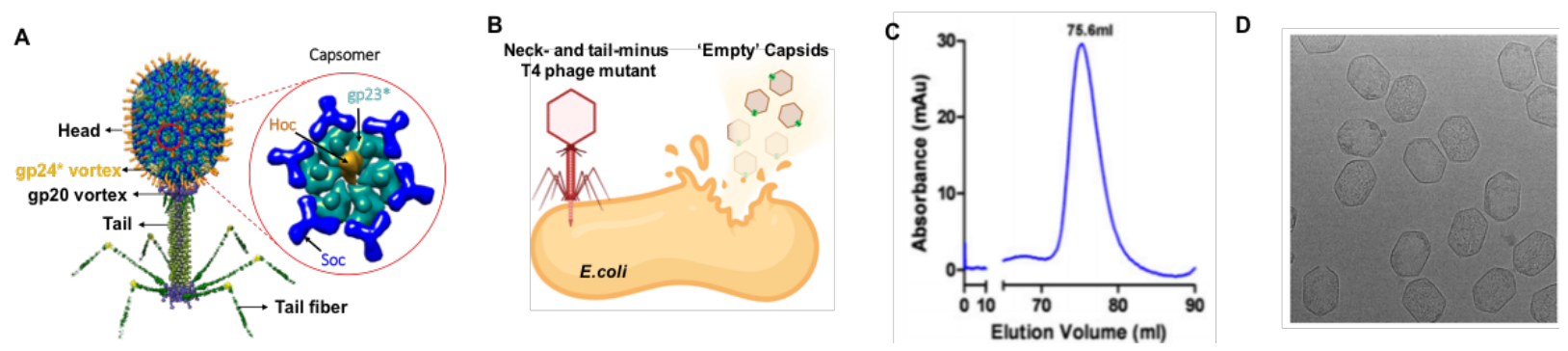

Fig S1: Purification of Hoc-Soc-T4 heads. A. Phage T4 Structure. The zoom in capsomer shows the major capsid protein gp23* (cyan) (the asterisk shows that it is the cleaved form) (930 copies), Soc (blue) (870 copies), and Hoc (yellow) (155 copies). B. Phage T4 capsids. T4 phage empty capsids can be purified by infection of non-suppressor E. coli with 10am.13am.Hocdel.Socdel phage. $\mathrm{C}$. The heads were prepared as described in Materials and Methods and separated through a step gradient of $\mathrm{CsCl}(0.26 \mathrm{mg} / \mathrm{mL}$ at the top layer and $0.93 \mathrm{mg} / \mathrm{mL}$ at the bottom layer $)$ and centrifuged at 40,000 rpm using SW55Ti rotor for $60 \mathrm{~min}$. The elution peak of heads, further purified by binding to DEAE-Sepharose ion-exchange chromatography (AKTA prime, GE Healthcare). The bound heads were eluted with a $\mathrm{NaCl}$ gradient, and the heads were eluted at about $200 \mathrm{mM} \mathrm{NaCl}$. The UV absorbance is plotted on the y-axis and elution volume on the x-axis. D. Cryo-electron micrograph of purified T4 heads. 

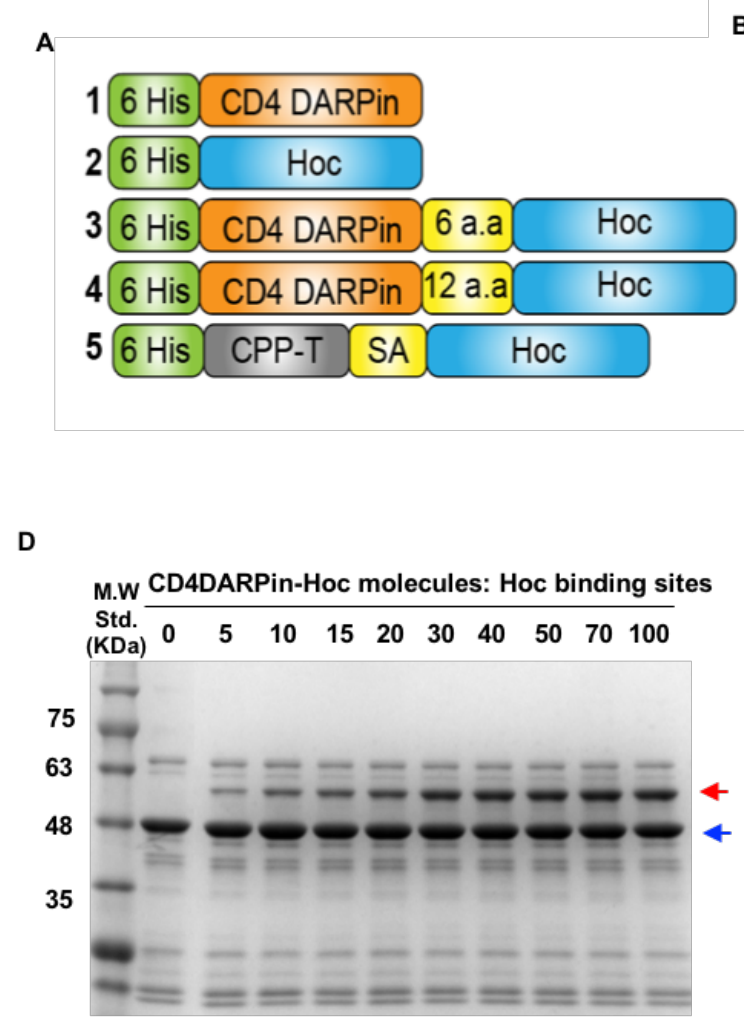
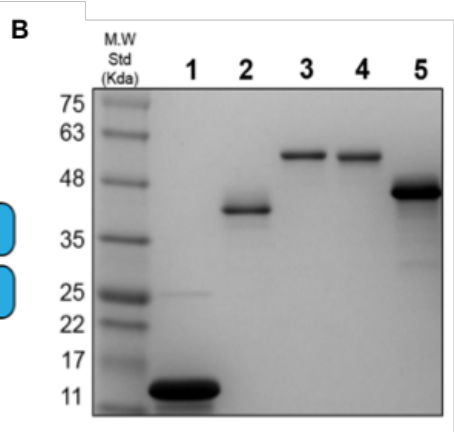

E

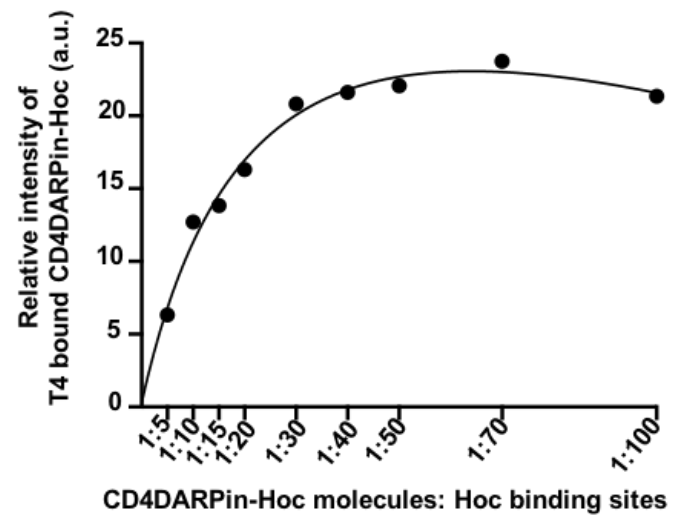

Fig S2: Construction, purification, and display of CD4DARPin-Hoc fusion proteins. A. Schematic representation of CD4DARPin (55.2)-Hoc fusion constructs. The DARPin coding sequence (orange) was fused to the N-terminus of Hoc (blue) via 6 a.a (LYKYSD) or 12 a.a (GGSGGSGGSGGS) linkers (yellow). The unfused CD4DARPin, Hoc and CPP-T-Hoc (grey) were prepared as controls. A hexa-histidine purification tag (green) was fused to the $\mathrm{N}$ terminus of all proteins. B. Reducing SDS-PAGE gel profiles showing bands of purified proteins. The recombinant proteins were overexpressed in E. coli cells and purified from the lysates by nickel affinity chromatography using HisTrap column followed by Hi-load 16/60 Superdex 200 gel filtration C. ELISA showing specificity of CD4DARPin-Hoc variants to soluble human CD4 receptor. Error bars show S.D and P-value was determined using two- tailed, unpaired t-test. **** $=\mathrm{P}$ value $<0.0001$. D. Display of CD4 DARPin-Hoc on the T4 surface at increasing ratios of CD4 DARPin-Hoc molecules to capsid Hoc binding sites (5:1 to 100:1). The red arrow shows position of bound CD4 DARPin-Hoc and the blue arrow shows position gp23 of capsids. E. CD4 DARPinHoc binding fit curve with nonlinear regression. The density volumes of T4-bound CD4DARPinHoc were quantified by laser densitometry based on the SDS-PAGE results shown in panel D. Similar results were obtained when the experiment was carried out with Hoc, CD4 DARPin-12a.aHoc, Hoc-T proteins. 


\section{CD4DARPinT4 -NPs efficiently target the CD4 receptor on CD4+ T cells}

The targeting ability of CD4DARPin-T4-NPs was analyzed by its ability to deliver packaged plasmids encoding reporter gene (Fig. 2A). Before delivery experiments, we determined whether CD4 DARPin-Hoc protein variants can recognize the CD4 receptor on the surface of T lymphocytes by using a modified cell-binding assay (Chand et al., 2017). For this assay, CD4DARPin test variants were coated on the wells in a 96-well plate and incubated with CD4positive T cells (A3.01), CD4-negative T cells (A2.01), and CD4 overexpressing (Tzmbl) cells. After washing off the unbound cells, the CD4DARPin-bound cells were quantified using a luciferase-based, CellTiterGlo detection. CD4DARPin variants bound efficiently to CD4-positive A3.01 and Tzmbl cells but not to CD4-negative A2.01 cells. On the other hand, CPP-T-T4 Hoc, a nonspecific cell binder bound to both CD4-positive and CD4-negative cells, and Hoc proteins used as a negative control showed no significant binding to any of the cells (Fig. 2B). CD4DARPin effectively blocked the binding of JRFL gp120 envelope protein (Env) to CD4+ T cells (A3R5 cells) when tested in a cell binding assay (Fig. 2C). These data demonstrate the specificity of binding of CD4DARPin-T4-NPs to CD4+ T cells in a manner similar to the binding of HIV-1 envelope protein (Schweizer et al., 2008).

To prepare CD4DARP-T4-NPs for targeted delivery of genes into CD4+ cells, the gp17 packaging ATPase motor protein was assembled at the portal vertex of T4 heads and the resultant packaging machine was used to package linearized pAAV_mCherry or pAAV_Luciferase plasmid DNAs in the presence of ATP. The packaging reactions were terminated by the addition of DNase I that degrades the unpackaged DNA. The encapsidated and DNase I-resistant DNA was released by treatment with proteinase $\mathrm{K}$ and quantified by agarose gel electrophoresis (Fig. 2D). CD4DARPin-Hoc was then displayed on the heads by adding it to the reaction mixture and the 
unbound protein was removed by centrifugation and washing with buffer (Fig. 2E). CD4DARPinHoc protein binding followed simple first-order kinetics and can be controlled by varying the ratio of CD4DARPin-Hoc protein molecules to Hoc binding sites. At a ratio of 30:1, nearly all of the 155 Hoc binding sites were occupied (Fig. S2, D \& E). Furthermore, the CD4DARPin-T4-NPs were able to target the delivery of the capsid-packaged eGFP and luciferase gene expressing plasmids into CD4+293T cells (Fig. 2F and 2G).

A

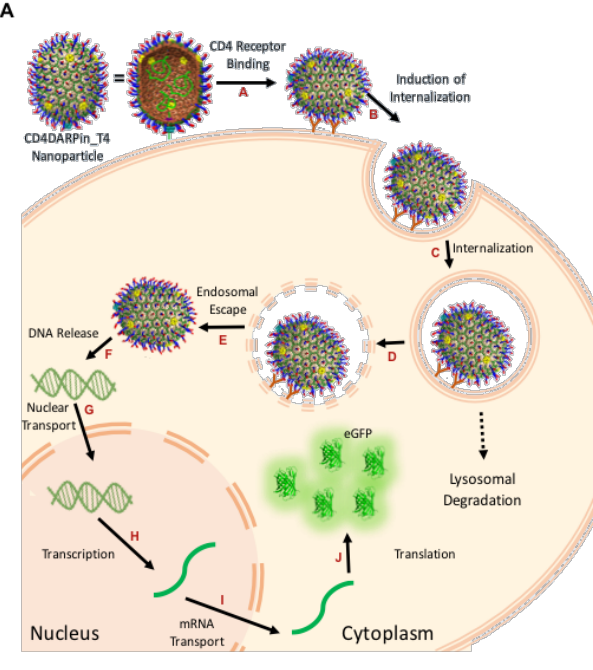

c
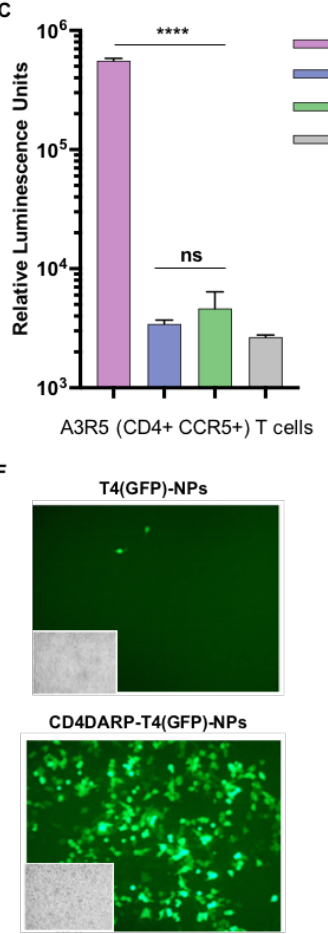

G

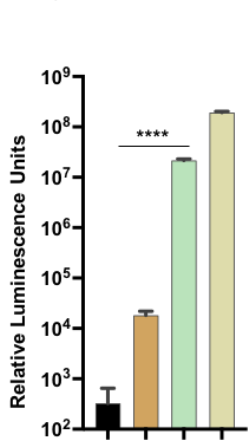

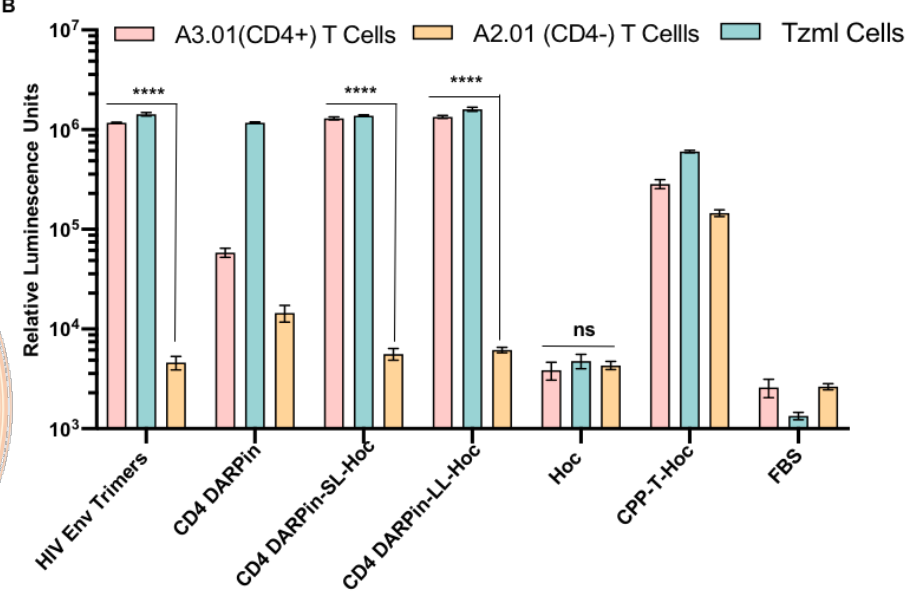

E
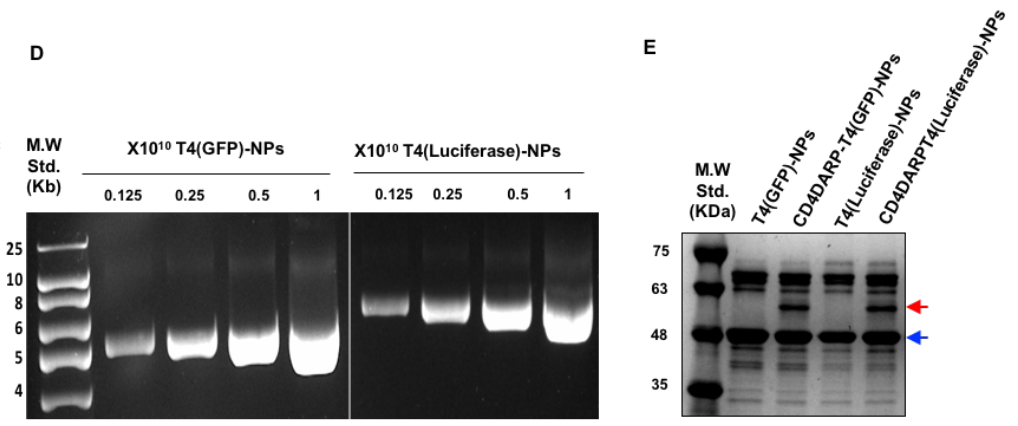

H

- Untranduced Cells

$\square$ T4(Luciferase)-NPs

$\square$ CD4DARP-T4(Luciferase)-NPs

$\square$ CPP-T-T4(Luciferase)-NPs



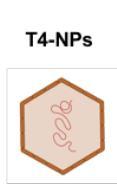

CD4DARP-T4-NPs
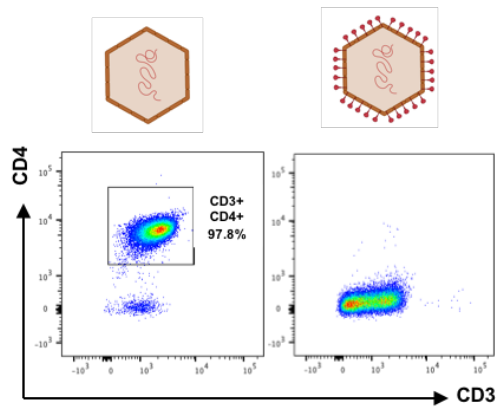
Fig 2. CD4DARP-T4-NPs efficiently target CD4 receptor on CD4+ $T$ cells. A. Targeted gene delivery by CD4DARP-T4-NPs. The NPs bind to cells through CD4 receptor and are internalized. The encapsidated DNA ( $g f p)$ is released into the cytosol. The DNA enters nucleus and undergoes transcription and expression of GFP. B. Specific binding of CD4 DARPin-Hoc to CD4 receptor on T cells. CD4DARPin Hoc variants, CD4 DARPin, Hoc, CPP-T-Hoc and HIV-1 gp140-JRFLTrimer were coated onto 96-well plate and blocked with FBS to prevent non-specific binding. The CD4+ (A3.01), CD4- (A2.01) and CD4 overexpressing (Tzmbl) cells were added to the wells and the extent of binding was quantified by luciferase- based CellTiterGlo Kit (Promega). Error bars show the S.D. P-value was determined using two-way ANOVA test. $* * * *=\mathrm{P}$ value $<0.0001 . \mathrm{C}$. CD4DARPin Hoc fusion protein blocked the binding of HIV Env Trimers to A3R5 cells (CD4+ $\mathrm{T}$ cells) in cell binding assay. A3R5 cells either untreated or treated with anti-CD4 antibody or CD4DARPin-Hoc were added to the wells and the extent of binding was quantified. D. DNA packaging in T4 capsids. Empty Hoc- Soc- phage T4 capsids were packaged with linearized eGFP or Luciferase reporter plasmid DNAs by assembly of gp 17 motor to the portal. Agarose gels show the amount of packaged DNA inside the T4 capsids. Using the energy from ATP hydrolysis the motor packages DNA molecules into the capsid. E. In vitro display of CD4DARPin-Hoc proteins. The red arrow shows position of bound CD4 DARPin-Hoc and the blue arrow shows position gp23 of capsids. F. eGFP DNA packaged capsids decorated with or without CD4DARPinHoc yield CD4DARP-T4(GFP)-NPs and T4(GFP)-NPs respectively and used for targeted delivery. Fluorescence and phase contrast micrographs of CD4+ 293T cells with CD4DARPT4(GFP)-NPs (Upper) and T4(GFP)-NPs (Lower). G. Expression of packaged and delivered luciferase gene quantified using luciferase assay. H. CD4DARP-T4-NPs efficiently bind CD4+ T cells in Human PBMCs. CD4+ T cells negatively isolated from human PBMCs were incubated with T4-NPs and CD4DARP-T4-NPs for 48 hours. Flow cytometry data shows that CD4+ T cells were undetectable with a CD4-specific antibody after treatment with CD4DARP-T4-NPs. Error bars show the S.D. P-value was determined using two- tailed, unpaired t-test. $* * * *=\mathrm{P}$ value $<0.0001$.

To determine the efficiency of targeting, the CD4DARP-T4-NPs were incubated with primary CD4 $\mathrm{T}$ cells negatively purified from human PBMCs followed by staining with fluorochrome-conjugated anti-CD3 and anti-CD4 mAbs. Nearly all the CD4+ T cells (97.8\%) could no longer be stained with anti-CD4 mAb when compared to the control cells treated with T4 heads lacking CD4DARPin (Fig. 2H). This experiment demonstrated that the CD4DARP-T4-NPs are highly efficient to target the CD4 receptor on human primary CD4 T cells that abrogated the binding of anti-CD4 mAb. To further confirm that the CD4DARPin-T4-NPs are associated with the cells, we performed an immunofluorescence assay by co-displaying the CD4DARPin-T4-NPs with eGFP-Soc and targeting these NPs to CD4 receptor-expressing 293T cells. Strong GFP 
fluorescence encircled the periphery of these cells but not with the control NPs (Fig S3 A,B,C,D,E

\& F) confirming the specificity of targeting of CD4DARP-T4-NPs to CD4+ cells.

A

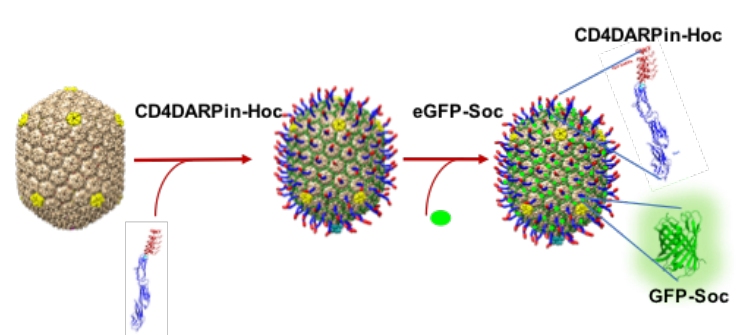

C
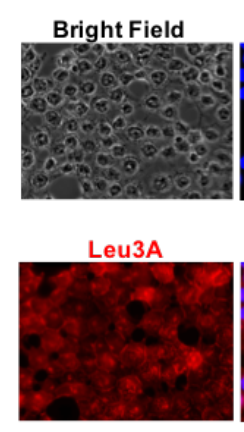

E
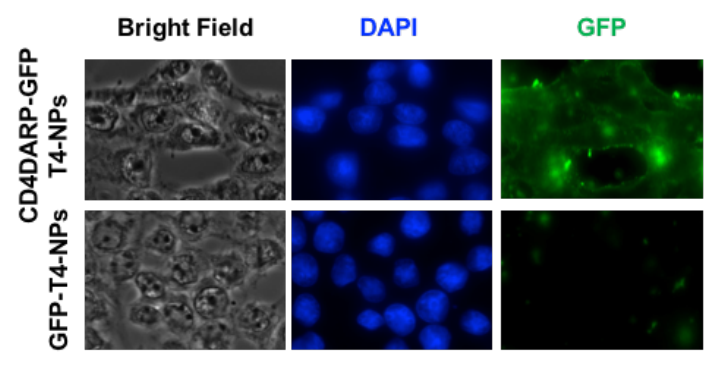

B
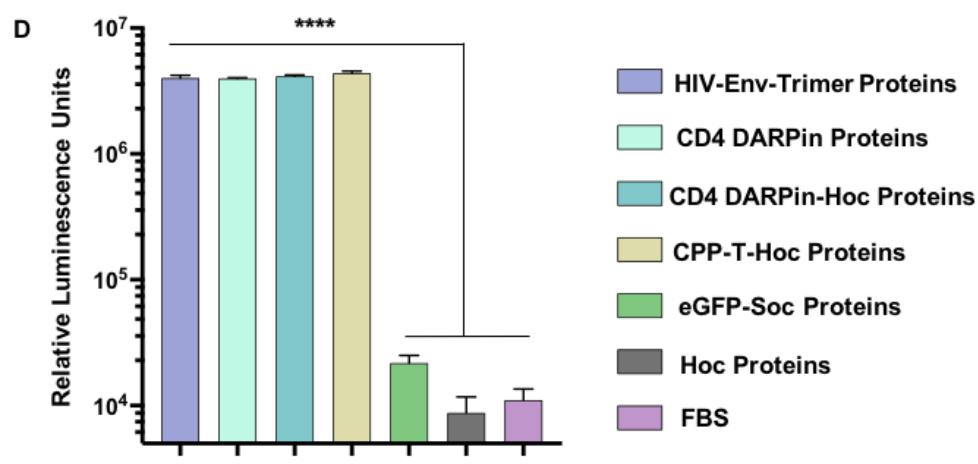

$\mathbf{F}$

GFP / DAPI

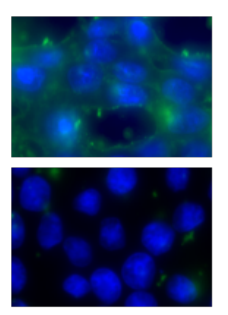

CD4DARP-GFP T4-NPS

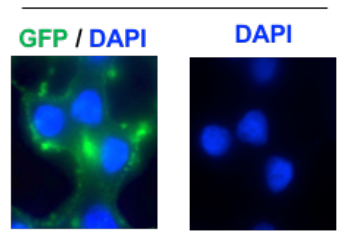

Fig. S3 Targeting of CD4DARPin-Hoc and eGFP-Soc co-displayed T4 heads. A. Schematic representation of CD4DARPin-Hoc and eGFP-Soc co-display. B) SDS-PAGE display gel profiles. About $4 \times 10^{10} \mathrm{Hoc}-$ Soc- heads were incubated with CD4DARPin-Hoc at 30:1 (CD4DARPinHoc molecule: Hoc binding sites) and increasing eGFP-Soc molecules to Soc-binding sites on the capsid (0:1-100:1), and display was carried out as described in Materials and Methods. After washing off the unbound protein, the samples were electrophoresed on a 4-20\% gradient SDSPAGE. The positions of the CD4 DARPin-Hoc protein (red) and eGFP-Soc (blue) are marked with arrows. C. CD4+ 293T cells were grown on coverslips and fixed by CASIO methods, followed by co-staining with anti-CD4 antibody, Leu3a(Red), and nucleus stain, DAPI (Blue). Red stain around the cell's periphery indicates the presence of a CD4 receptor on the surface D. Specific binding of CD4 DARPin-Hoc to CD4 receptor on CD4+ HEK293T cells by cell binding assay as described before. E. CD4+293T cells were grown on coverslips and fixed by CASIO methods, followed by incubating with either CD4DARPin-Hoc displayed or CD4DARPin-Hoc and eGFP co-displayed T4-NPs for 60 mins, followed by washing and fixing with DAPI-mount media and 
observation with fluorescence microscopy. Images are 60X magnification. D. GFP/DAPI merged image at $100 \mathrm{X}$.

\section{The T4-NPs activate innate immune cells and Tymphocytes in human PBMCs}

Next, we sought to determine whether the T4-NPs could activate human T cells. Freshly isolated human PBMCs from healthy donors were left unstimulated or were incubated with T4NPs or the CD4DARP-T4 -NPs. CD3/CD28 mAbs and/or PHA were used as positive controls for PBMCs activation. The activation status was determined by surface expression of CD25 and CD38, which were well-documented as late and early $\mathrm{T}$ cell activation markers respectively, by flow cytometry (Fig S4, A). CD25 is the alpha-chain of the IL-2 receptor and needs the beta-chain of the IL-2 receptor for the execution of IL-2 signaling (Ross and Cantrell, 2018). CD38 is another well-characterized marker of immune activation in HIV infection that prolongs the proliferation and survival of CD4+ T cells (Glaria and Valledor, 2020).

CD38 surface expression in T4-NPs and CD4DARP-T4-NPs treated cells showed the activation of innate immune cells (CD3- T cells), which include NK cells and dendritic cells (Fig. 3A). A significant increase in surface expression of CD25 and CD38 was observed with T4-NPs and CD4DARP-T4-NPs, though profoundly less than the global activation shown by CD3/CD28 mAbs in CD3+ cells (Fig. 3B \&C). Furthermore, profiling for secreted Th-1 (IL-2, IFN- $\gamma$, IL-10, TNF$\boldsymbol{\alpha})$ and Th-2 (IL-4, IL-5, IL-12, IL-13) cytokines in cell supernatants reflected the immune cell activation by phage T4-NPs (Fig. 3 D \& E). Activation was observed in total CD3+ T cells and gated CD4+ and CD4- T cells, which represents the basal level of activation associated with T4NPs. The observed activation of innate cells might indicate the innate to the adaptive direction of immune activation. The observed basal activation by T4-NPs might be due to the packaged doublestranded DNA containing unmethylated $\mathrm{CpG}$ motifs that are known to stimulate innate immune 
responses by interacting with Toll-like receptors (TLRs) such as TLR9 expressed on the endosomal surface of the antigen-presenting cells (Iwasaki and Medzhitov, 2004).
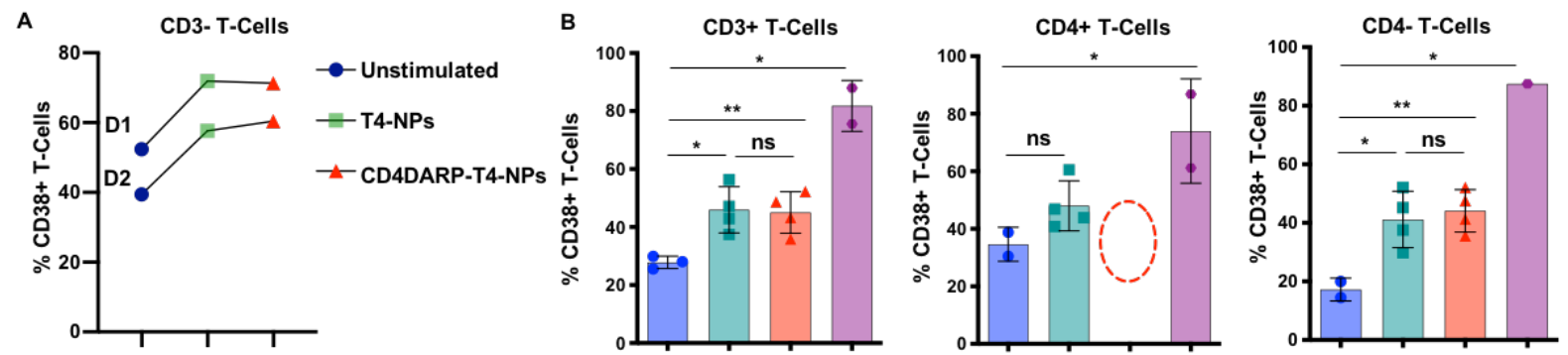

C
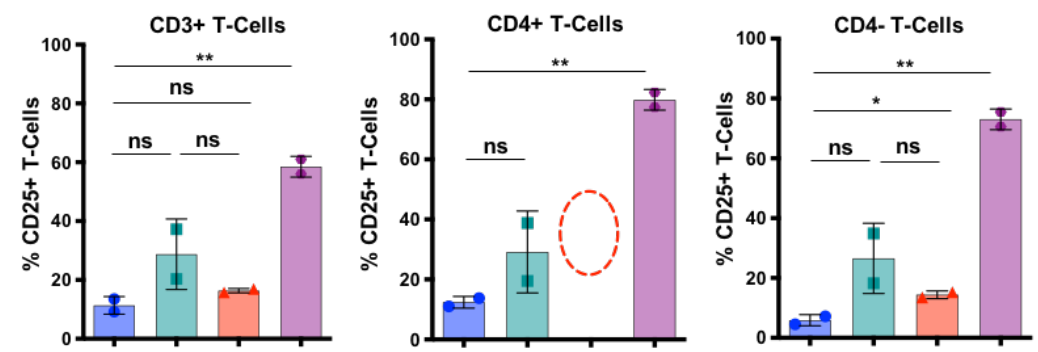

- Unstimulated (Negative Control)

D
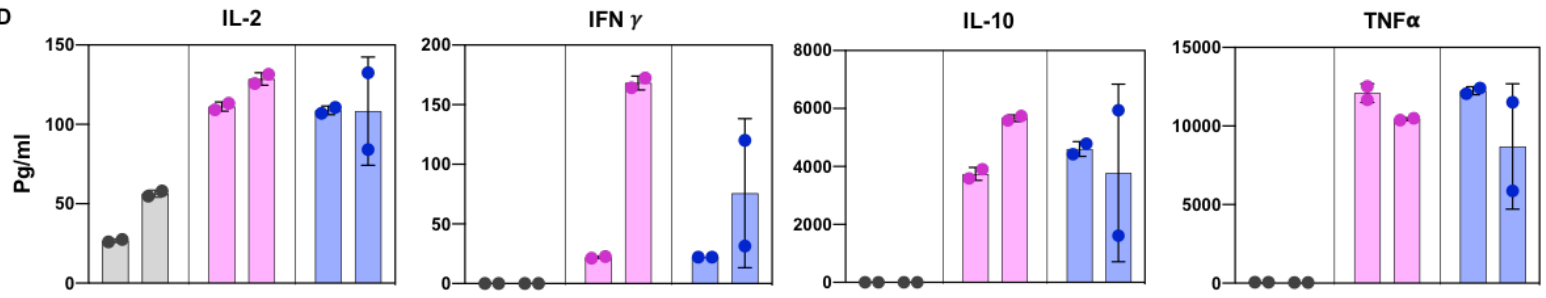

$E$
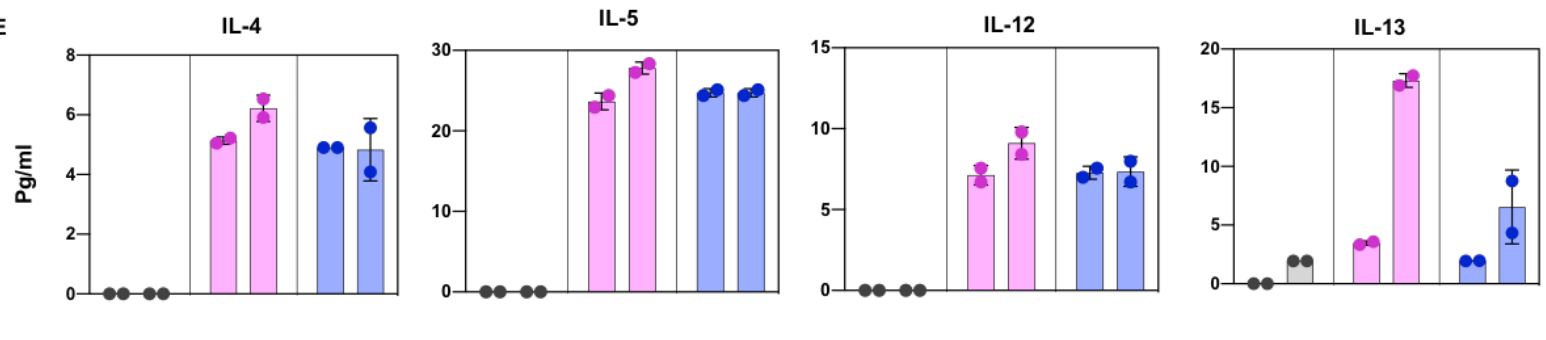

- Unstimulated

- T4-NPs

- CD4DARP-T4-NPs

Fig 3. The T4-NPs activate innate and adaptive $T$ lymphocytes in human PBMCs. PBMCs from healthy human donors were left unstimulated or were stimulated with the T4-NPs, CD4DARP-T4-NPs, and anti-CD3 \& CD28 mAbs. After 48 hours of incubation, cells were costained for CD3, CD4 receptor, anti-CD25, and anti-CD38 and analyzed by flow cytometry. A. CD38 surface expression in CD3- T cells (D1 \& D2 represents two different donors) B. CD38 surface expression in CD3+ T cells C. CD25 surface expression in CD3+ T cells. The Cytokines quantification from supernatants was done with Bio-Plex Pro ${ }^{\mathrm{TM}}$ Human Cytokine Th1/Th2 Assay D. Th1 cytokines- IL-2, IFN- $\gamma$, IL-10, TNF- $\alpha$ E. Th2 cytokines- IL-4, IL-5, IL-12, IL-13. Data are mean $\pm \mathrm{SD}$. Two-tailed unpaired t-test, $* *=\mathrm{P}$ value $<0.005, *=\mathrm{P}$ value $<0.05$ 
A

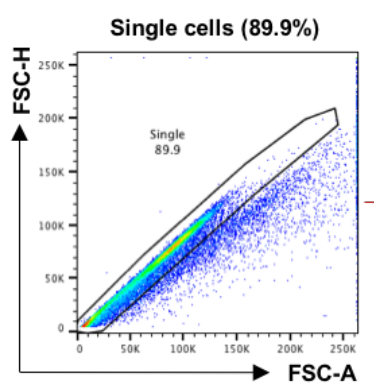

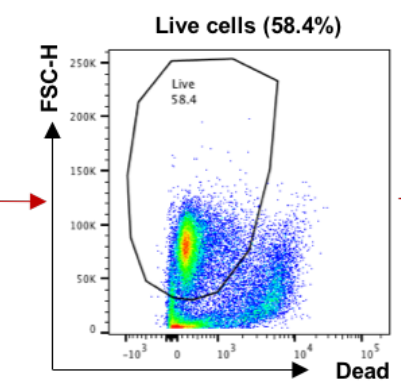
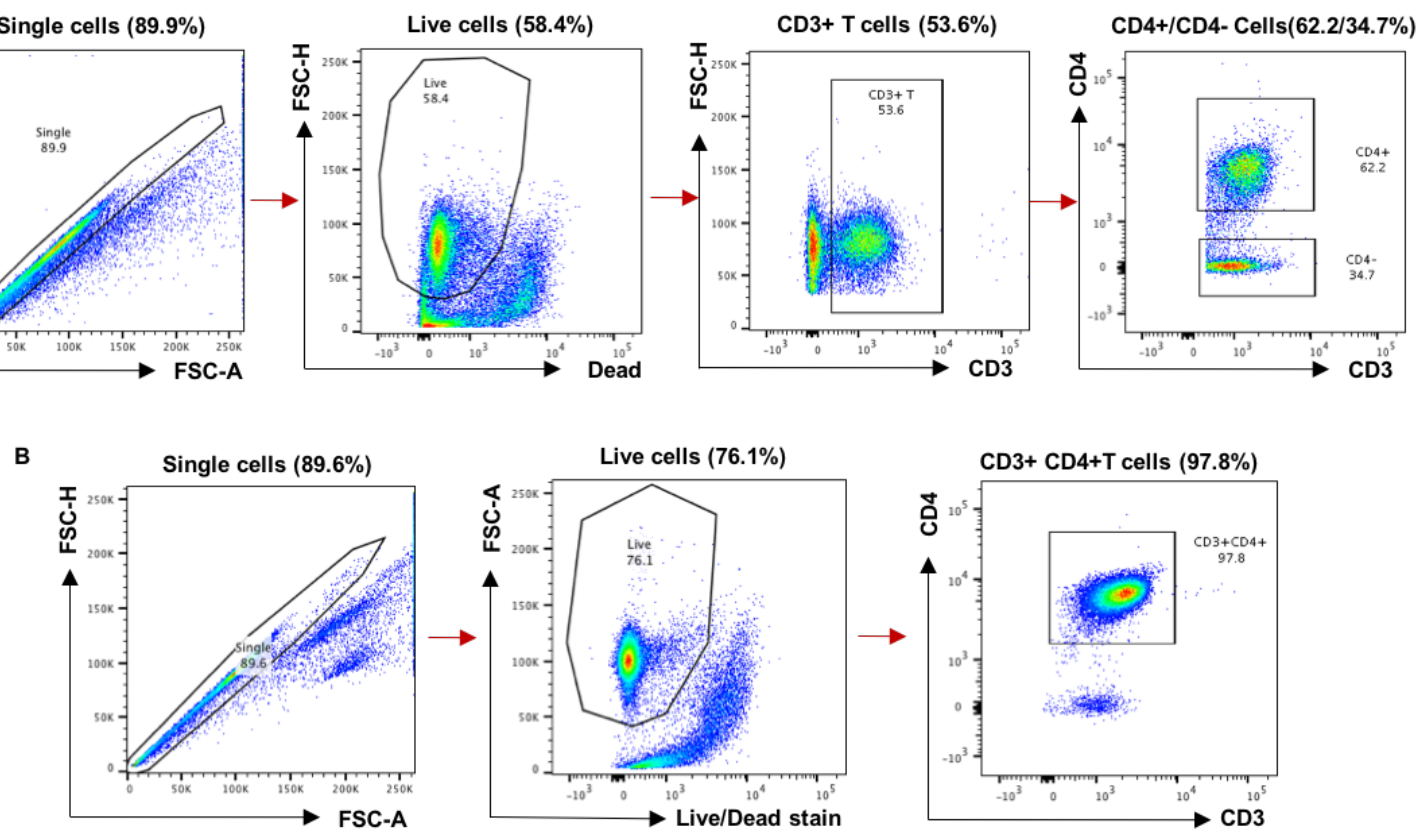

Fig. S4. Flow cytometry plots detailing gating strategy. A. PBMCs from healthy human donors were left unstimulated or were stimulated with the T4-NPs, CD4DARP-T4-NPs, and anti-CD3 \& CD28 Abs. After 48 of incubation, cells were co-stained for CD3, CD4 receptor, anti-CD25, and anti-CD38 and gated for flow cytometry analysis as shown in Fig.3. B. Confirmation of purification of CD4+ CD25- resting T cells from healthy human donors.

\section{CD4DARP-T4 -NPs activate (not globally) primary resting CD4+ T cells}

Host responses to a viral vector may induce susceptibility of vector-specific T cells to HIV infection by upregulation of the HIV-1 co-receptor, CCR5 (Auclair et al., 2018). The potential effects of T4-NPs on the susceptibility of vector-specific CD4+ T cells to HIV infection was evaluated by analyzing the surface expression of CCR5 in PMBCs by flow cytometry. We found that neither T4-NPs nor CD4DARP-T4 -NPs altered CCR5 expression in PBMCs (Fig. 4A). CD4+ T-cells represent the major cell population of HIV latent reservoir. To determine if CD4DARPT4-NPs can activate resting CD4+ T-cells, these cells from healthy human donor PBMCs were isolated by negative selection and their purity was established by flow cytometry (97.8\% CD3+CD4+ T-cells) (Fig. S4, B). The purified cells were then either left unstimulated or were 
stimulated with T4-NPs and CD4DARP-T4-NPs. CD3/CD28 mAbs and PMA mediated stimulations were used as positive controls. After 48 hours of incubation, CD38 surface expression was analyzed to determine the activation status of resting CD4+ T cells. We found no significant increase in CD38 expression in T4-NPs treated cells while CD4+ T cells were undetectable due to the targeted binding of CD4DARP-T4-NPs (Fig. 4B). Nevertheless, a characteristic clustering of the cells was observed under the microscope, in cells treated with CD4DARP-T4-NPs and antiCD3/Cd28 mAbs or PMA (positive controls) but not with the T4-NPs treated cells or the untreated cells (negative control) (Fig.4C, upper panel). To characterize the observed phenotypic activation, we determined the surface expression of CD25, a global activation marker. No significant increase in CD25 expression was observed. However, about $25 \%$ of the cells increased in size when treated CD4DARP-T4-NPs, as evident from the dot-plots. As expected, anti-CD3/Cd28 mAbs activated cells showed a major increase in CD25 surface expression (Fig. 4C, lower panel). Similar phenotypical clustering and the increase in cell size have been reported previously when CD4+ T cells were treated with human immunodeficiency virus-1 (HIV-1) envelope protein gp120 that binds with human CD4 with high affinity (Kinet et al., 2002). 
A

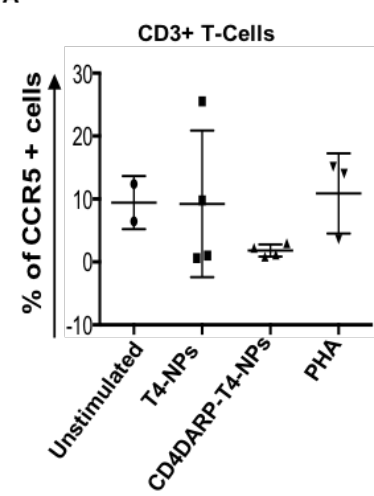

C
B
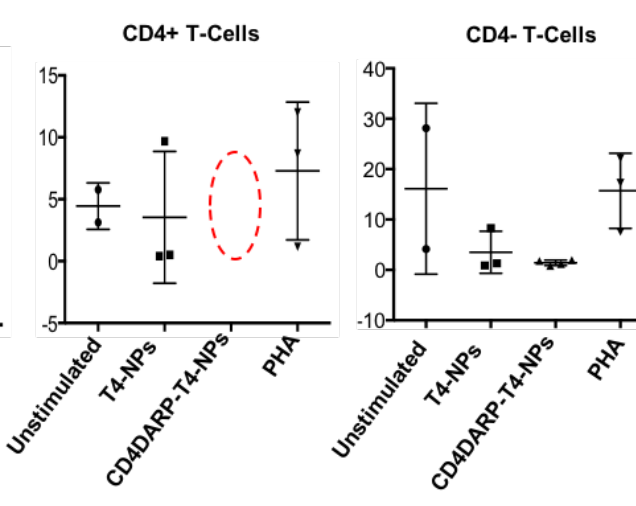

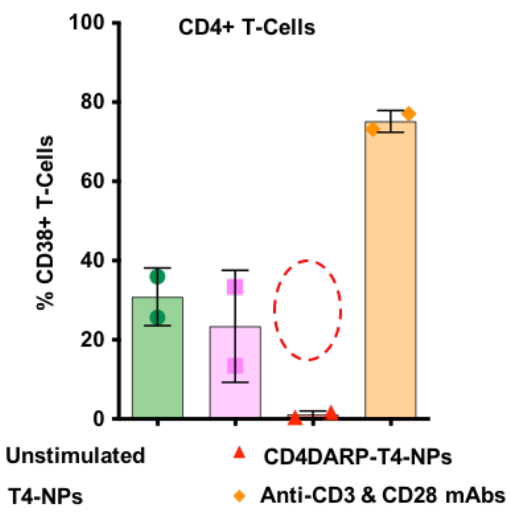

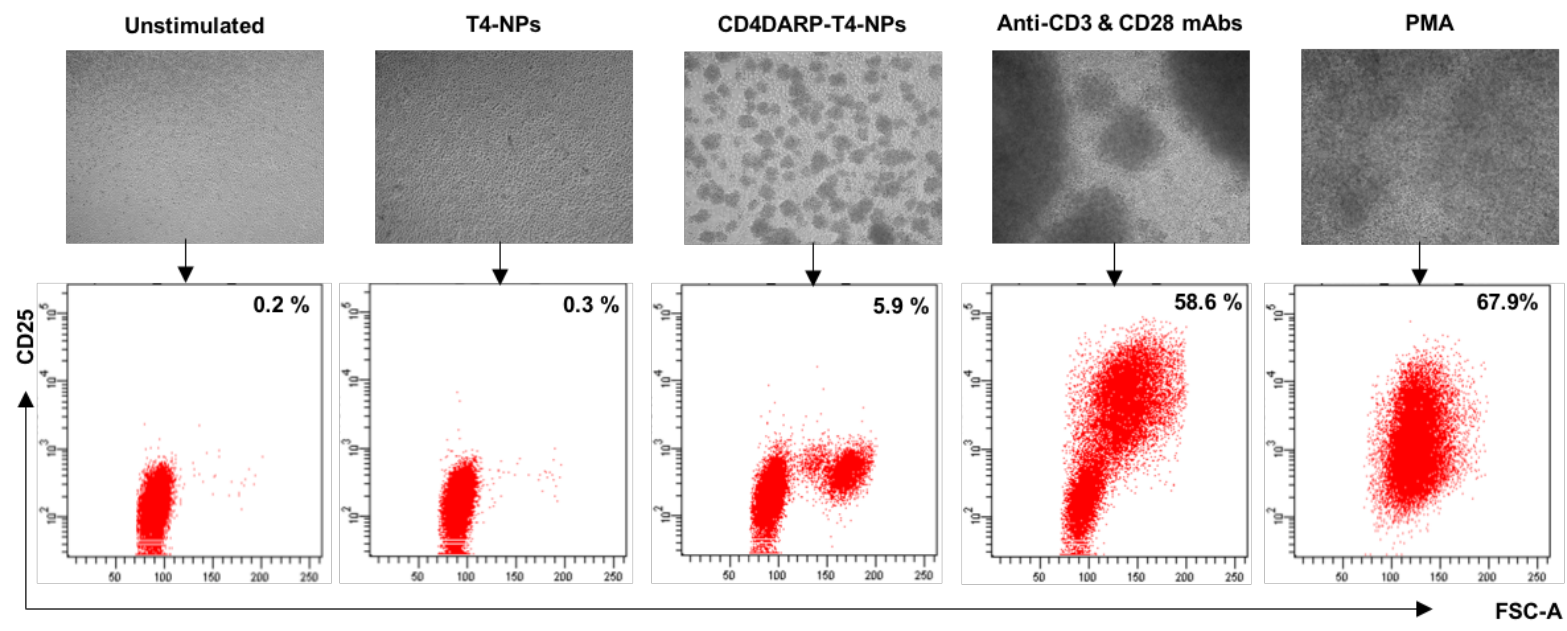

D
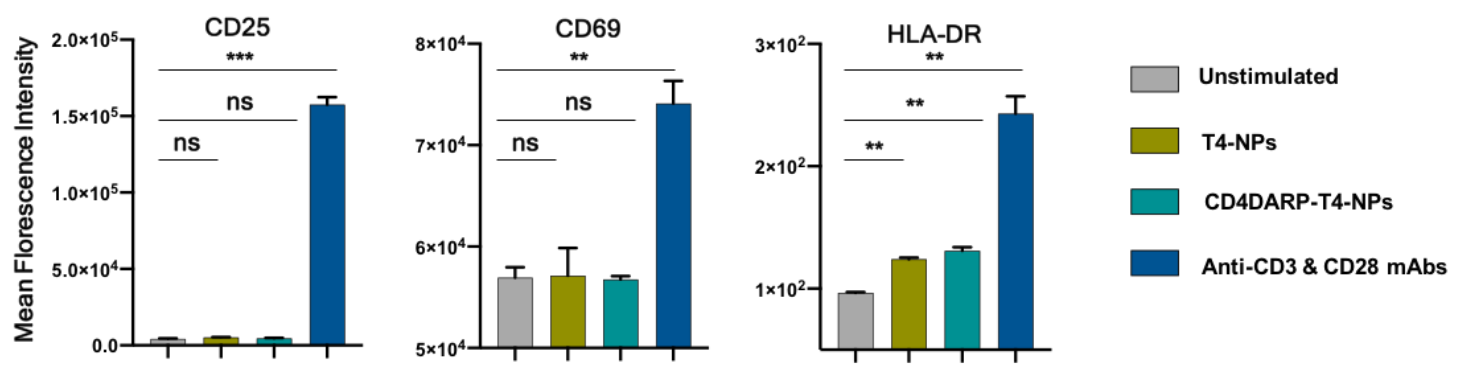

Fig 4. CD4DARP-T4 -NPs activate (not globally) primary resting CD4+ T cells. PBMCs from healthy human donors were left unstimulated or were stimulated with the T4-NPs, CD4DARP-T4NPs, and anti-CD3 \& CD28 mAbs. After 48 of incubation, cells were co-stained for CD3, CD4 receptor, anti-CCR5 Abs and analyzed by flow cytometry. A. CCR5 surface expression. B-D. CD4+ CD25- resting T cells were isolated by negative selection from human PBMCs and were left unstimulated or were stimulated with the T4 -NPs, CD4DARPin T4 -NPs, anti-CD3 \& CD28 mAbs and/or PHA. After 48 hours of incubation B. CD38 expression in purified CD4+T cells. C. Microscopic images (10X magnification) showing cell clustering as an activation phenotype (upper panel). Cells from all the groups were stained with FITC anti-CD25 mAbs and analyzed by flow cytometry. C. Quantification of surface expression of activation markers was done by costaining with conjugated mAbs, CD25-PE, CD69-APC, and HLA-DR-FITC and analyzed by flow 
cytometry. Data are mean $\pm \mathrm{SD}$. Two-tailed unpaired t-test, $* * *=\mathrm{P}$ value $<0.001 * *=\mathrm{P}$ value $<0.005$ $* *=\mathrm{P}$ value $<0.005, *=\mathrm{P}$ value $<0.05$

We have also determined the surface expression of other activation markers such as CD25, CD69, HLA-DR, and CD38 in the activated CD4+ CD25- resting T cells isolated from healthy donors PBMCs by flow cytometry to probe the activation mechanism of this unexpected observation. No significant increase in the expression of any of these global $\mathrm{T}$ cell activation was observed in either T4-NPs or CD4DARP-T4-NPs while both the positive controls showed substantial increases in the surface expression of these activation markers (Fig. 4 D). Similarly, the Th1 or Th2 specific cytokines were also not detected in culture supernatants of cells stimulated with either T4-NPs or CD4DARP-T4 -NPs (Fig. S5 A\&B). The above results demonstrated that CD4DARP-T4-NPs did not cause global T cell activation.

A

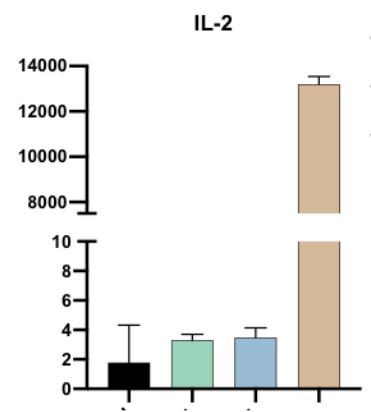

B

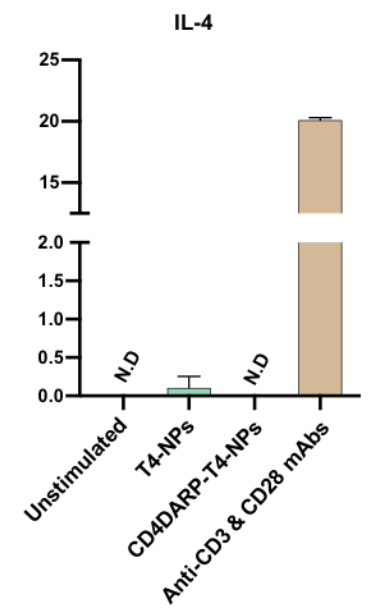

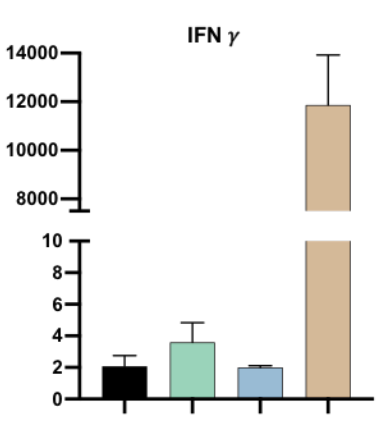

IL-5

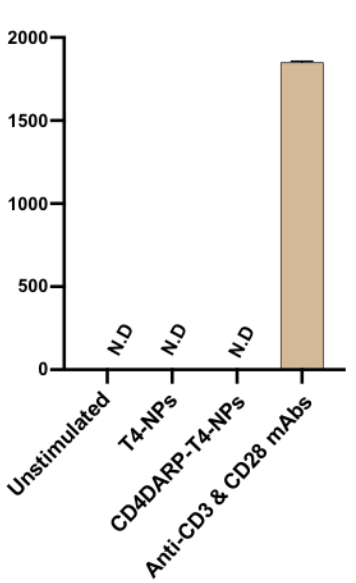

IL-10

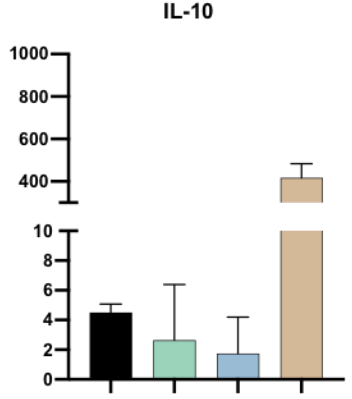

IL-12

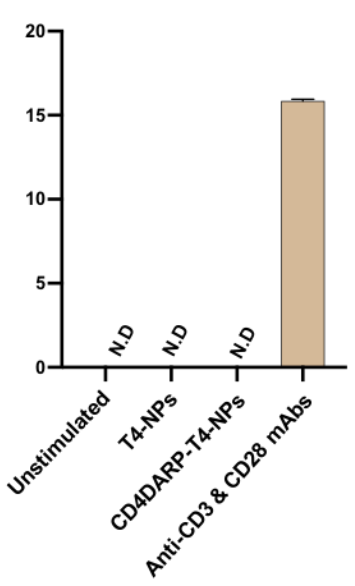

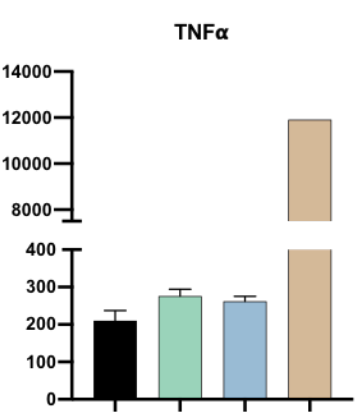

IL-13

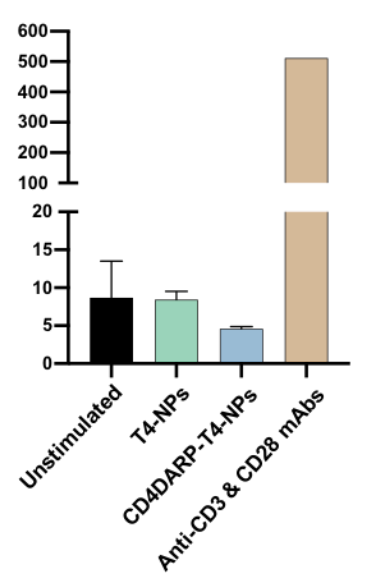


Fig S5. The T4-CD4DARPin -NPs do not elicit TH1 and TH2 cytokine responses from primary resting CD4+ T cells freshly isolated from human PBMCs. Freshly isolated CD4+ CD25- resting $\mathrm{T}$ cells by negative selection from human PBMCs were left unstimulated or stimulated with the T4 -NPs, CD4DARPin T4 -NPs, and anti-CD3 \& CD28 mAbs. After 48 hours of incubation, cytokines quantification was done with Bio-Plex Pro ${ }^{\text {TM }}$ Human Cytokine Th1/Th2 Assay A. Th1 cytokines- IL-2, IFN- $\gamma$, IL-10, TNF- $\boldsymbol{\alpha}$ B. Th2 cytokines-IL-4, IL-5, IL-12, IL-13. Data are mean $\pm \mathrm{SD}$ of duplicate samples.

\section{CD4DARP-T4-NPs and HIV-Env-T4-NPs activate in vitro cell model of latent HIV-1 infection}

We used J-Lat 10.6 full-length cells, a transformed cell line model of HIV-1 latency to determine reactivation of HIV proviruses by T4-NPs. The J-Lat cell line was originally developed by infecting Jurkat cells with a full-length HIV-1 vector having a frameshift mutation in the env gene and the $g f p$ gene replacing nef. These cells don't produce virus or viral proteins and are GFPnegative, indicating that they are transcriptionally silent and in the post-integration latency state. Therefore, expression of viral marker GFP would indicate reactivation of HIV in the J-Lat cells (Jordan et al., 2003).

Two types of CD4 targeted phage T4 -NPs were prepared, one displayed with CD4DARPin fused to Hoc and another with a recombinant CRF_AE HIV-1 gp140 (T/F100) fused to Soc (Ananthaswamy et al., 2019). HIV-1 gp140-Soc fusion protein construct was expressed in HEK293F cells and purified by Strep-Tactin affinity chromatography followed by size-exclusion chromatography (Fig. S6, A \& B). A series of biochemical analyses were performed after purification, such as Blue native PAGE, reducing and non-reducing PAGE to determine the proportion of trimers, nonspecific crosslinking and aggregation, and extent of furin cleavage (Fig. S6, C \& D). In addition, antigenicity analysis was done using PGT145 bNAb, which recognizes conformational epitopes at the apex of the closed trimer (Fig. S6, E). Being a quaternary Ab, PGT145 binds preferentially to the cleaved trimer in "closed" state but not to the "open" uncleaved trimers (Walker et al., 2011). The 8ANC195 Ab was used as a control as it binds to both open and 
closed trimers. We found that the gp140-Soc fusion protein binds to the mAbs at similar efficiency as the gp140, suggesting that gp140-Soc retained a "native-like" conformation (Fig. S6, F).

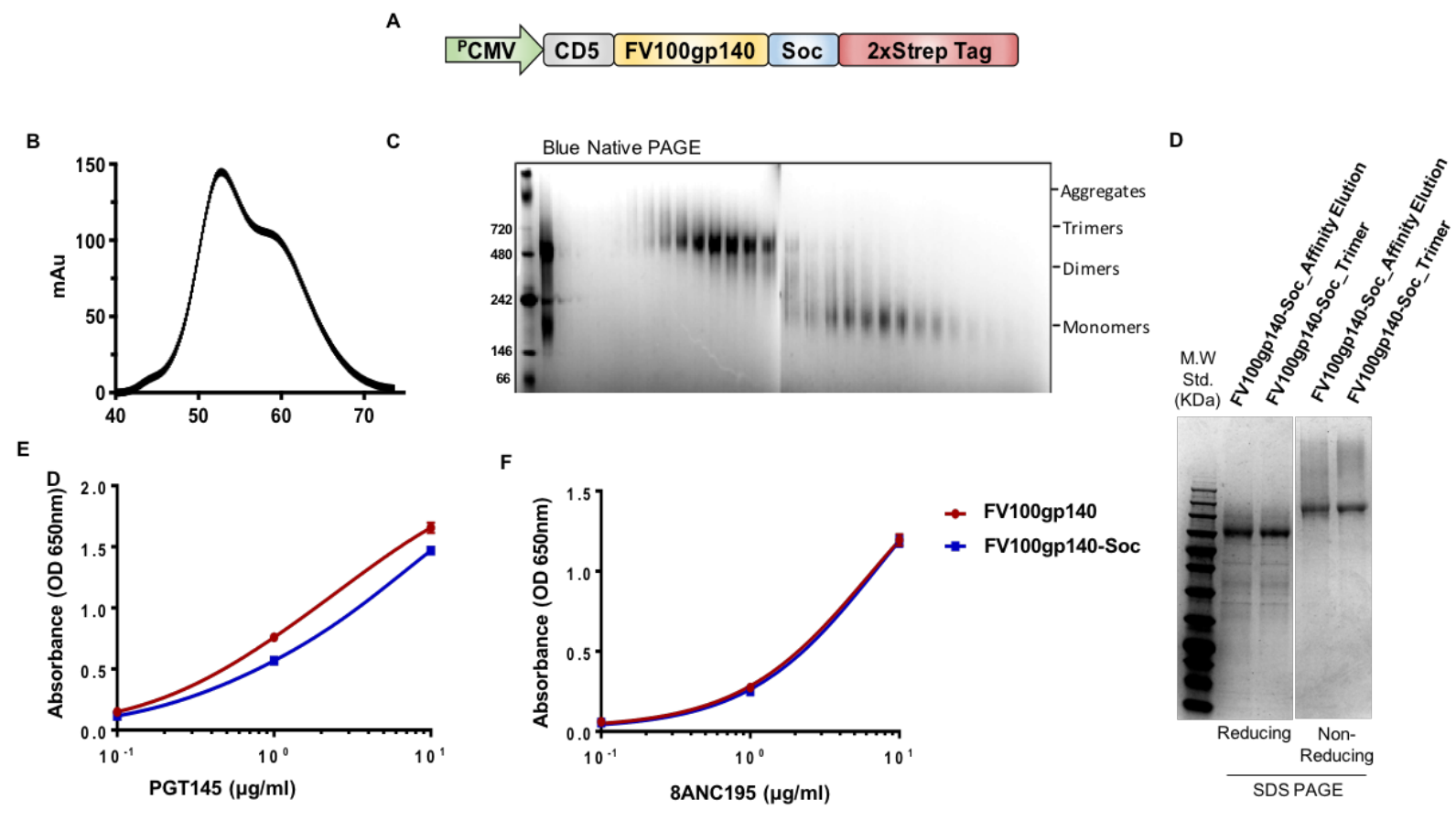

Fig. S6 Purification, Biochemical analyses and antigenicity of gp140-soc trimers. A. Construct design of gp140-soc fused trimers. B. size-exclusion chromatography (SEC). C. Blue-NATIVE gel profiles (non-denaturing) of eluted fractions after SEC are shown for) FV100-soc. D. Reducing and non-reducing SDS-PAGE of affinity chromatography-purified sample and size-exclusion chromatography trimeric fraction. E \&F. Antigenicity of gp140-soc fusion trimers. Comparative binding of purified cleaved gp140 and gp140-soc trimers is depicted with trimer specific mAbs 8ANC195 and PGT145. Binding curves are color coded for the respective trimer, shown in the key provided on the right side of the graph. Trimers were captured on the Strep-Tactin ELISA plates through the C-terminal Strep-Tag II at the constant concentration of $1 \mu \mathrm{g} / \mathrm{ml}$ for the assay. Each panel shows the binding curve from three replicates at the indicated concentrations of the mAbs. No statistically significant difference in binding was observed between gp 140-soc fusion and native gp140 trimer pairs at $10 \mu \mathrm{g} / \mathrm{ml}$ of the mAbs tested using unpaired two-tailed test.

The T4-NPs, CD4DARP-T4-NPs and HIV-Env-T4-NPs, were incubated with J-Lat cells. After 48 hours, we found that the phage T4 capsid by itself showed low but significant activation of HIV-1 when compared to the untreated control as quantified by flow cytometry (Fig. 5, A\&B). This result is consistent with the earlier observation that the T4-NPs can induce low levels of 
activation of human PBMCs and also T cells in general. This activation could be due to the surface structure of T4 capsid consisting of repetitive and symmetric disposition of capsid protein subunits that mimics the PAMPs (pathogen associated molecular patterns) present on human viral pathogens, or the residual $\sim 8 \mathrm{~kb}$ CpG phage DNA (TLR9 agonist) remaining inside the T4-NPs used in these experiments. However, the CD4DARP-T4-NPs that targeted the CD4 receptor showed far greater levels of J-Lat cell activation, 23.5\% compared to 9.9\% with the T4-NPs. Furthermore, soluble (undisplayed) CD4DARPin Hoc or JRFL HIV-1 gp140-Soc proteins at the same copy number did not show significant activation. These observations suggest that the CD4 receptor-ligand displayed T4-NPs by mimicking the HIV-1 virion bound to J-Lat cells potentially through multipoint interactions that then led to efficient reactivation of the transcriptionally silent HIV provirus. This targeting specificity was demonstrated by several control experiments. First, CD4DARPin-Hoc and HIV-1 gp140-Soc fusion proteins bound specifically to J-Lat cells but not to CD4- T cells (A2.01) and PMA treated J-Lat cells which is known to downregulate the CD4 surface expression (Pelchen-Matthews et al., 1993) (Fig. S7, A). Second, T4-NPs co-displayed with CD4DARPin-Hoc and mCherry-Soc but not mCherry-soc alone stained the J-Lat cells on the periphery (Fig. S7, B). Third, CD4DARP-T4-NPs activate J-Lat cells in a dose and time-dependent manner as determined by the expression of viral GFP by fluorescence microscopy (Fig. S8 A\&B). 
A

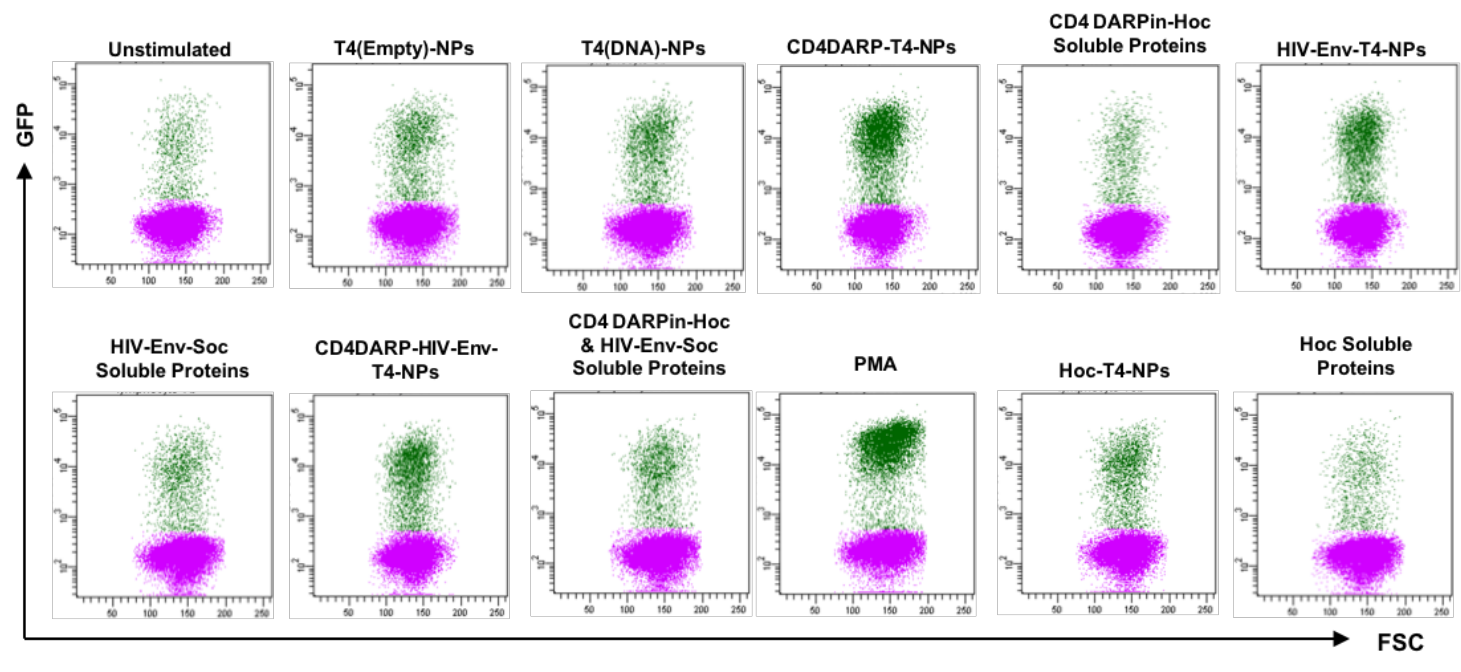

B GFP Fluorescence

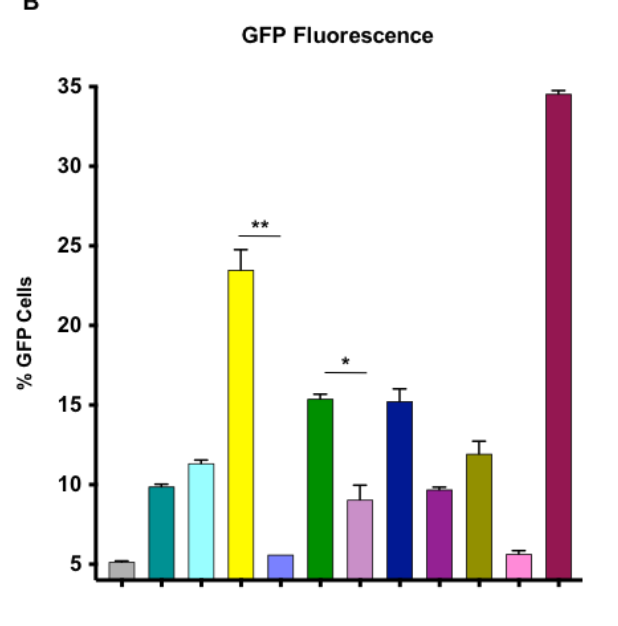

c

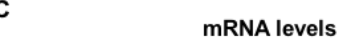

$\mathbf{F}$
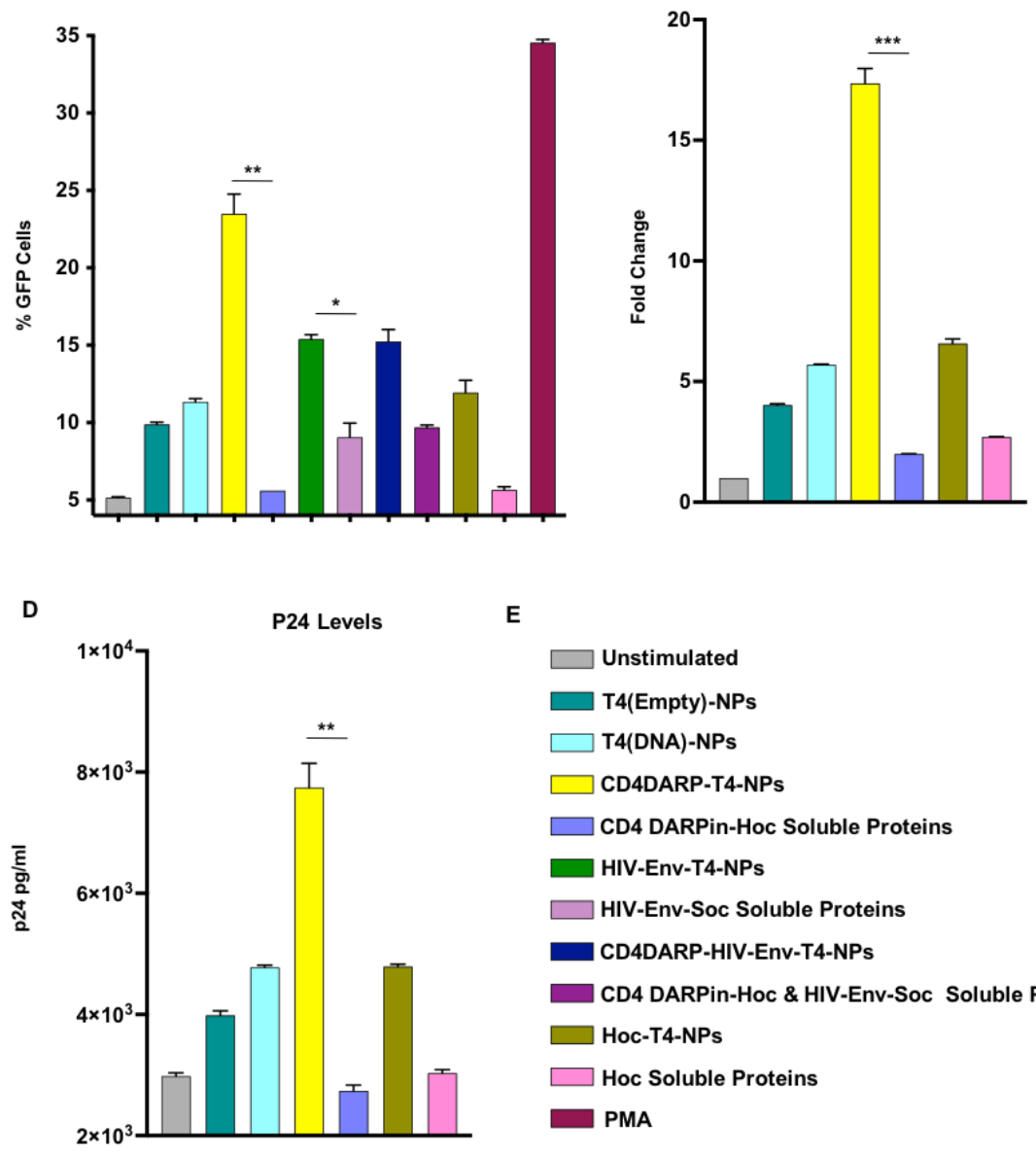

E
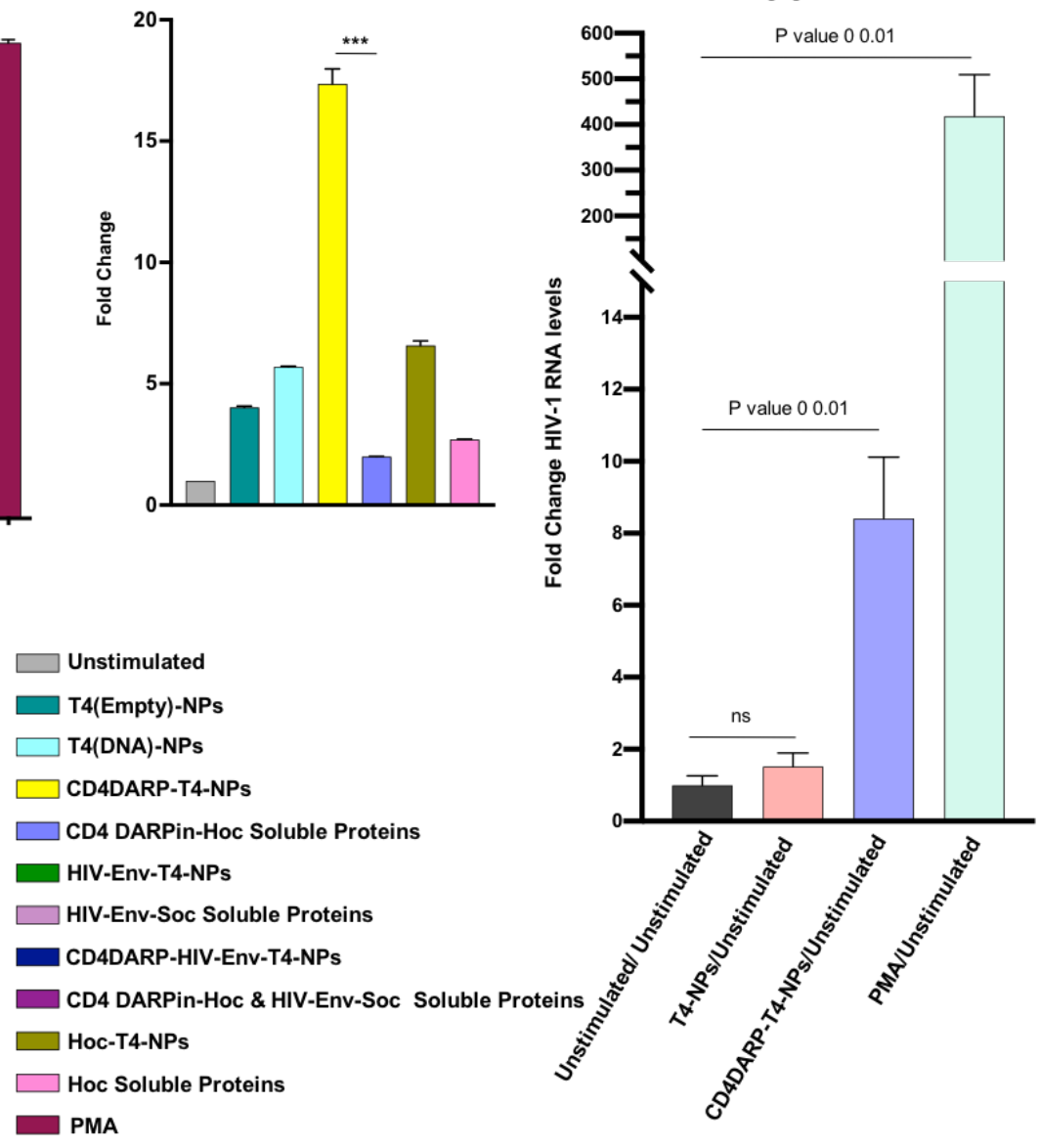

Fig 5. CD4DARPin-T4 -NPs mediated J-Lat 10.6 activation. A. Flow cytometry plots profiling GFP expression in J-Lat cells when incubated with T4-NPs, CD4DARP-T4-NPs and controls NPs, and proteins. Green and magenta dots in plots represents GFP positive and GFP negative cells respectively. X-axis represents intensity of GFP positive cells and Y-axis represents cell size (FSC) 
B. Quantification of flow cytometry plots shown in panel A. Data are means \pm S.D. of duplicate samples and represent two independent experiments C. Viral mRNA levels quantified using real time RT-PCR and normalized to beta-actin mRNA levels. The results were compared to unstimulated samples and showed as fold change. Data are means \pm S.D of duplicate samples D. Gag-derived p24 HIV protein in the cell culture supernatant assayed using an p24 ELISA. Average of three measurements \pm S.D are shown. E. legends for panel B, C and D. F. HIV-1 Genomic RNA levels in the cell culture supernatant assayed by real-time RT-PCR with RCAS RNA as an internal control. The results are represented as fold change from unstimulated samples. Data are means \pm S.D. of duplicate samples and represent two independent experiments.

A

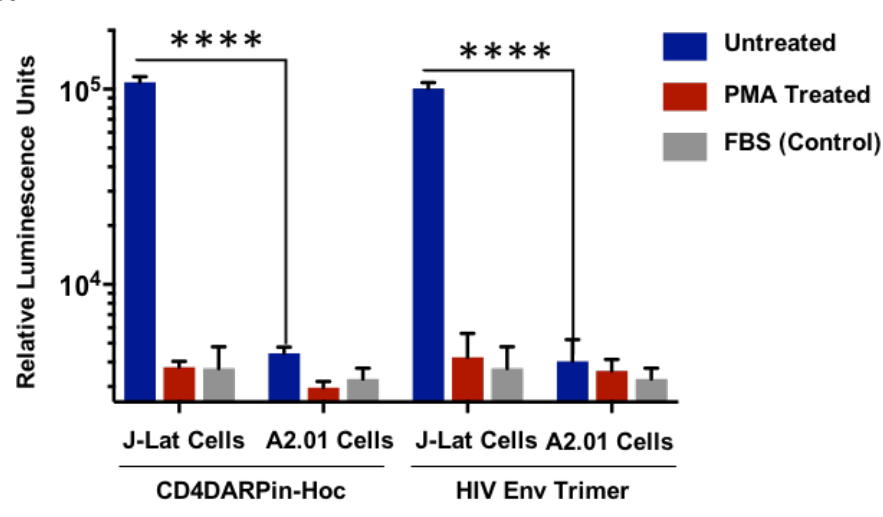

B

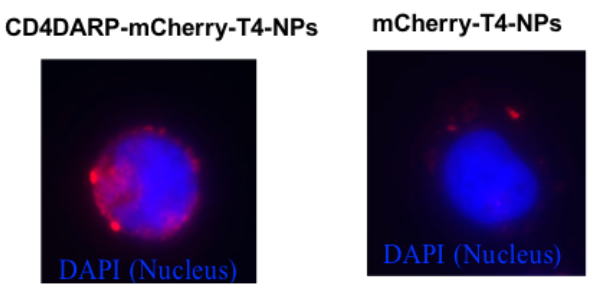

Fig S7: Targeting of CD4-DARPin-Hoc and mCherry-Soc co-displayed T4 heads. A. CD4 DARPin Hoc and HIV-1 gp140-JRFL-Trimer were coated onto a 96 well plate and blocked with FBS to prevent non-specific binding. The J-Lat 10.6 full length cells were added to the wells and the extent of binding was quantified by the chemiluminescence - based assay (CellTiteGlo Kit, Promega) B. J-Lat 10.6 Full length cells were fixed on coverslips by CASIO methods (see materials and methods for details) followed by incubating either CD4DARPin-Hoc displayed or CD4DARPin-Hoc and mCherry-Soc co-displayed T4 -NPs for 60 mins followed by washing and fixing with DAPI-mount media and observed with fluorescence microscopy. Images are $60 \mathrm{X}$ magnification. P-value was determined using two- tailed, unpaired t-test. $* * * *=\mathrm{P}$ value $<0.0001$. 
A

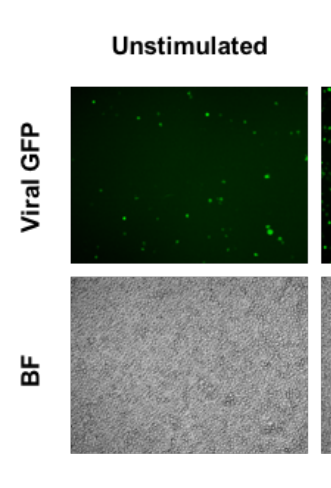

B
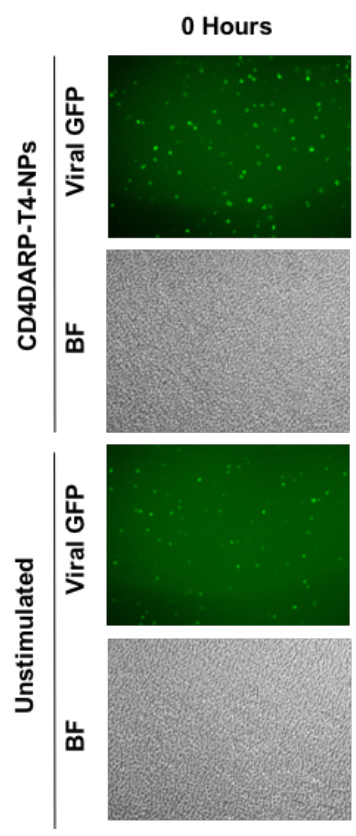

T4-NPs
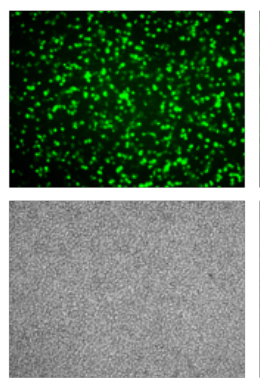

0 Hours
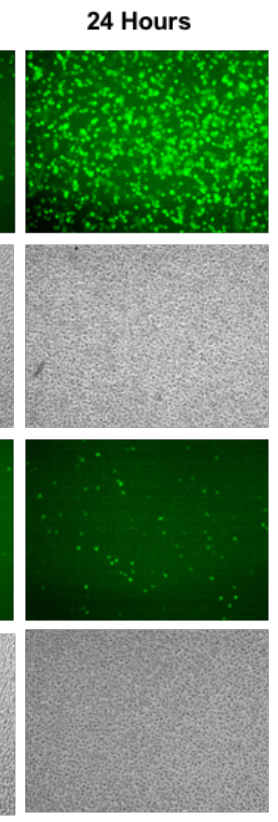

CD4DARP-T4-NPs
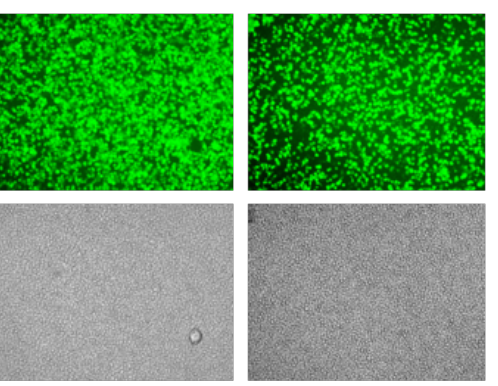

48 Hours
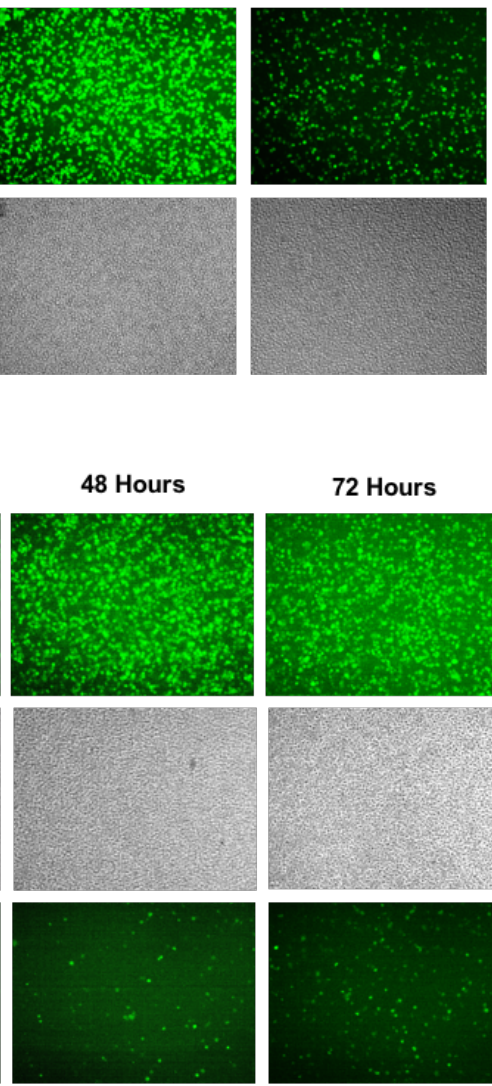

PMA
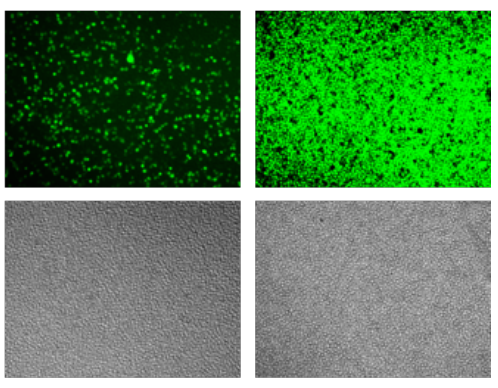

Fig S8. The effects of CD4DARP-T4 -NPs on the reactivation of latent HIV-1 in J-Lat cells. A. The dose-response of CD4DARP-T4 -NPs on reactivation of latent HIV-1 in J-Lat cells. The effect of T4 -NPs was determined by expression the GFP using fluorescence microscopy. B. The time dependent reactivation of CD4DARP-T4 -NPs in reactivation of latent HIV-1 in J-Lat cells. The effect of T4 -NPs was determined by expression the GFP using fluorescence microscopy.

\section{CD4DARP-T4 -NPs reverse latency in the in vitro cell model of latent HIV-1 infection}

Although the viral genome's GFP expression is an indicator of provirus reactivation, to confirm the effect of phage T4 -NPs on the latent HIV-1, HIV-1 transcripts levels were examined by qPCR. The qPCR results correlated with GFP expression; the viral transcription was increased to 5.69-fold by T4-NPs while CD4DARP-T4-NPs enhanced the transcription by 17.4-fold 
compared to the unstimulated cells (Fig. 5 C). These results suggest transcriptional activation of the resident HIV-1 latent provirus in cells. To further analyze the assembly and release of HIV-1 virions, the presence of HIV-1 virions in the culture supernatant were quantified by p24 ELISA. About 1.6-fold increase in HIV gag/p24 antigen was detected by T4-NPs while CD4DARP-T4NPs enhanced it by 2.6-fold compared to unstimulated cells (Fig. 5, D).

Recently, it has been shown that latently infected cells can release viral proteins such as HIV-1 Gag protein without the actual viral particle release in an in vitro HIV model (Pace et al., 2012). Therefore, it was necessary to determine if phage T4-NPs release the HIV-1 viral particles, not just the HIV Gag protein. To distinguish these two, the culture supernatant from the activated cells was concentrated and the viral RNAs were isolated. The copy number of HIV-1 genomic RNAs was determined by multiplex qPCR using a primer-probe set for HIV-1 gag and RCAS gag (internal control). An 8-fold increase in the HIV-1 RNA levels was observed in the culture supernatant of cells treated with CD4DARP-T4-NPs compared to supernatants of unstimulated cells (Fig. 5 F).

Together, the above results suggest that J-Lat cell activation leads to proviral transcription, and assembly and release of HIV-1 virions following the treatment with CD4DARP-T4-NPs.

\section{CD4DARP-T4 -NPs activate latent HIV-1 independent of PKC and NFAT pathways}

Physiologically, CD4 assists T-cell Receptor (TCR)'s communication with Antigen Presenting Cells (APCs). Specifically, CD4 receptor binds to MHC-II on the APCs mediating downstream signaling that ultimately leads to T-cell activation. $\mathrm{PKC} \theta$ is a major regulator in TCR signaling pathways (Isakov and Altman, 2002). Therefore, we asked whether CD4DARP-T4-NPs act via PKC signaling pathway to activate the latent HIV-1 in J-Lat cells. Using the pan PKC 
inhibitor Gö6983, we found that PKC inhibition completely suppressed PMA-induced activation of latent HIV-1 and reduced the anti-CD3 and anti-CD28 co-stimulatory response by $\sim 50 \%$. However, no effect on the activation of latent HIV-1 by CD4DARP-T4 -NPs was observed upon treatment with Gö6983 (Fig. 6A). These data suggest that CD4DARP-T4-NPs do not act via the PKC signaling pathway to activate the latent HIV-1.

NFAT, another essential transcription factor involved in the T cell activation, is also known to reactivate HIV-1 gene expression (Kinoshita et al., 1997). To assess the possibility of NFAT playing a role in J-lat activation, we used cyclosporin A (CsA), an inhibitor of the NFAT signaling pathway. We found that CsA treatment did not suppress the CD4-targeted CD4DARP-T4-NP reactivation of latent $\mathrm{HIV}-1$, while it did inhibit the effect of ionomycin that activates $\mathrm{T}$ cells via NFAT pathway (Fig. 6B).

The above results further demonstrate that the CD4DARP-T4-NPs mediated activation is not global activation as the PKC and NFAT pathways are two critical pathways involved in global T cell activation.
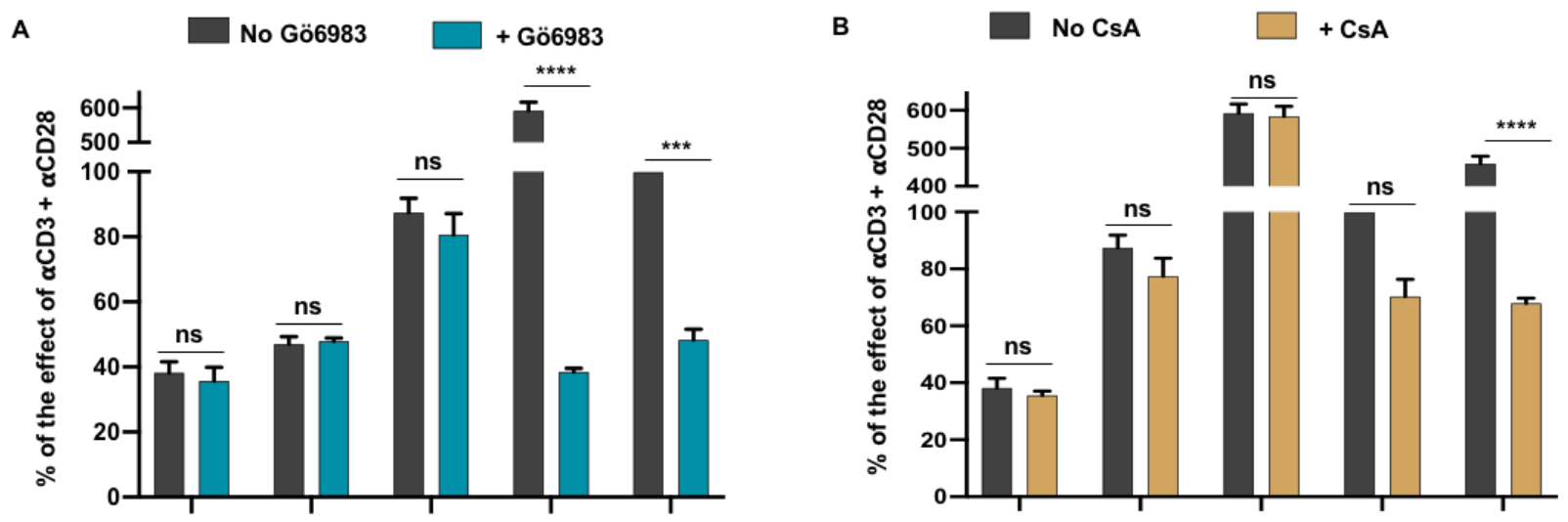

Fig. 6 CD4DARP-T4 -NPs activate latent HIV-1 independent of PKC and NFAT pathways. A.PKC independent activation. Effect of a PKC inhibitor on the reactivation of latent HIV-1 by CD4DARP-T4-NPs. J-Lat 10.6 full-length cells were incubated with PKC inhibitor Gö6983 for 1 hour prior to the treatment with each activator. Cells were collected and analyzed for GFP-positive 
cells after 48 hours of incubation. The results were normalized to the effect of anti-CD3 plus anti$\mathrm{CD} 28$ co-stimulation. Data are mean $\pm \mathrm{SD}$ of duplicate samples from a representative experiment. B. NF-AT independent activation. Effect of CsA on the reactivation of latent HIV-1 by CD4DARPT4-NPs. J-Lat 10.6 full-length cells were incubated with CsA 1 hour prior to the treatment with each activator. Cells were collected and analyzed for GFP-positive cells after 48 hours of incubation. Data are mean $\pm \mathrm{SD}$ of duplicate samples from a representative experiment. P-value was determined using two- tailed, unpaired t-test. $* * * *=\mathrm{P}$ value $<0.0001, * * *=\mathrm{P}$ value $<0.001$.

\section{DISCUSSION}

HIV remains a significant global health problem as nearly 38 million people worldwide are currently living with HIV-1 and $\sim 2$ million new infections occur every year, but there is neither a vaccine nor a cure. The "shock-and-kill" strategy for HIV cure postulates that reactivation of virus transcription in CD4+ T cells, containing stably integrated, intact, and transcriptionally silent forms of HIV-1 proviruses is the first essential step to eliminate the latent reservoir in ART-treated people living with HIV. How to specifically activate these reservoir cells without causing global activation remains a major hurdle for clinical implementation of this strategy. Here, we present observations that show the potential of engineered bacteriophage T4 nanoparticles to target the CD4+ T cells and induce a degree of HIV proviral reactivation without leading to global T cell activation.

HIV-1 uses multivalent display of its envelope glycoprotein to efficiently bind and activate CD4+ T-cells. In this study, we created an HIV-1 viral mimic by displaying the CD4 ligand, CD4 DARPin, fused to Hoc on the capsid surface of a bacterial virus, the phage T4. We demonstrated, both by ELISA and cell binding assays that such CD4 DARPin-T4-NPs bind to CD4 receptor with high affinity and exquisite specificity. These nanoparticles emanating Hoc molecules at symmetrical positions of the capsid, with the CD4DARPin positioned at the tips of $\sim 170 \AA$ flexible fibers are well-positioned to capture the CD4 receptor on T cells. 
The efficient targeting ability of CD4DARPin-T4-NPs was demonstrated in two ways. First, the delivery of packaged reporter genes-expressing plasmids into T cells resulted in $\sim 67120$ fold increase in luciferase activity when compared to control T4-NPs lacking the CD4DARPinHoc display. Second, remarkably, CD4+ T cells in human PBMCs were titrated out after treatment with CD4DARP-T4-NPs and hence became undetectable by a CD4-specific antibody. Indeed, the eGFP- or mCherry-Soc co-displayed CD4DARPin-T4-NPs generated a layer of fluorescence around the CD4+ cells demonstrating the targeting specificity of the phage T4 HIV-mimic.

Our data demonstrate that both the T4-NPs and CD4DARPin-T4-NPs increase the surface expression of CD38 activation marker in both CD3+ T-cells and CD3- non-T cells which include adaptive T cells and innate cell types, respectively. Presumably, T4-NPs efficiently activate PBMC through innate cell types such as dendritic cells and macrophages. T4-NPs mediated activation could be explained by the presence of residual $\sim 8 \mathrm{~kb}$ DNA present in the capsid. It was recently reported that phages can mediate the activation of the human immune system through interaction of $\mathrm{CpG}$ in genomic DNA with TLR9 in the endosomes of macrophages, which when co-cultured with CD4+ T cells cause the activation (Gogokhia et al., 2019). The activation of PBMCs by T4NPs was also reflected by the release of Th1 and Th2 cytokines.

Perhaps a more important observation is that the CD4DARPin-T4-NPs or the NPs displayed with the HIV-1 gp140 trimers caused robust proviral reactivation in J-Lat cells as determined by a series of assays including reporter GFP expression, increased proviral transcription, and release of assembled virions by the activated cells. This observation is consistent with the finding that implicated in the activation of CD4+ T cells by calcium-mediated signaling upon gp120 binding without necessarily involving viral entry (Perez et al., 1991). CD4 receptor on $\mathrm{T}$ cells plays a critical role in enhancing TCR mediated T cell activation by interacting with 
MHC class II molecules on antigen presenting cells (APCs). The CD4 receptor, on its cytoplasmic domain, interact with the src family tyrosine kinase p561ck which is involved in initiating TCR signaling. CD4 blockade by a soluble or membrane-bound gp120 is shown to be associated with activation of CD4+ T cells.

Notably, however, activation by the phage T4 CD4DARPin-NPs does not lead to global T cell activation. This is an important distinction because many of the current shock-and-kill reagents investigated for HIV-1 cure lead to massive global activation that might lead to anaphylactic shock. This was evidenced by two observations. First, activation of J-lat cells does not activate PKC or NFAT pathways as demonstrated by the inhibitors of these pathways do not affect CD4DARPinT4 mediated activation. PKC was documented to be a master regulator of multiple signaling pathways such as AP-1, NF- $\mathrm{BB}$ and ERK1/2. Also, NFAT mediated pathways are well documented in $\mathrm{T}$ cell activation. Bypassing the PKC $\theta$ and NFAT pathways may allow CD4DARPT4-NPs to avoid the detrimental full-blown T cell activation.

The CD4DARP-T4 NPs activation was also recapitulated in primary CD4+ T cells freshly isolated from human PBMCs. The cells showed no significant increase in the global activation markers such as CD69, CD25 and HLA-DR. But phenotypically, cells treated with CD4DARP-T4 NPs showed clustering and increase in size. This phenotype has been shown previously to be associated with activation of T cells (Vasiliver-Shamis et al., 2009). The clustering phenotype was also seen in ART suppressed patient derived CD4+ T cells when treated with CD4DARP-T4-NPs which suggest some kind of activation in CD4+T cells, irrespective of HIV-1 infection, which is not global.

In conclusion, we showed that the engineered phage T4 nanoparticles serve as HIV-1 viral mimics and exhibit remarkable targeting specificity to $\mathrm{CD} 4+\mathrm{T}$ cells. In addition to delivering 
genes, such targeting leads to $\mathrm{T}$ cell activation in a way that does not lead to global activation and cytokine storm. It also leads to proviral HIV-1 genome reactivation and assembly and release of new virion particles in a HIV-latency model cell line but not in patient derived primary T cells. The targeted and selective activation of $\mathrm{T}$ cells hold promise for potential development of an effective HIV cure strategy and novel immunotherapeutics in the future.

\section{MATERIALS AND METHODS}

Purification of 10-amber13-amber hoc (highly antigenic outer capsid protein)-del.soc (small outer capsid protein)-del Heads.

The 10-amber 13-amber hoc-del soc-del T4 phage mutant was constructed by standard genetic crosses and mutant heads were purified according to previously described protocols (ref). briefly. E. coli $\mathrm{P} 301$ (sup-) cells $(500 \mathrm{~mL})$ infected with this mutant were lysed in $40 \mathrm{~mL}$ of Pi-Mg buffer (26 mM Na2HPO4/68 mM NaCl/22 mM KH2PO4/1 mM MgSO4, pH 7.5) containing 10 $\mu \mathrm{g} / \mathrm{mL}$ DNase I and chloroform $(1 \mathrm{~mL})$ and incubated at $37^{\circ} \mathrm{C}$ for $30 \mathrm{~min}$. The lysate was subjected to two low-speed $(6,000 \times \mathrm{g}$ for $10 \mathrm{~min})$ and high-speed $(35,000 \times \mathrm{g}$ for $45 \mathrm{~min})$ centrifugations, and the final heads pellet was resuspended in $200 \mu \mathrm{L}$ of Tris $\cdot \mathrm{Mg}$ buffer $(10 \mathrm{mM}$ Tris $\cdot \mathrm{HCl}, \mathrm{pH}$ 7.5/50 $\mathrm{mM} \mathrm{NaCl} / 5 \mathrm{mM} \mathrm{MgCl} 2$ ) and purified by $\mathrm{CsCl}$ density gradient centrifugation. The major head band sedimented at about 1/3 from the bottom of a 5-mL gradient was extracted and dialyzed overnight against Tris $\cdot \mathrm{Mg}$ buffer. The heads were further purified by DEAE-Sepharose chromatography (ref). The peak heads fractions were concentrated and stored at $-80{ }^{\circ} \mathrm{C}$.

\section{Cell lines and reagents}

J-Lat full-length clone 10.6, ACH-2, A3.01, A2.01 and Tzmbl cells were obtained through NIH AIDS Research and Reference Reagent Program. Human PBMC, StemCell Technologies 
Inc., CA (Catalog \# 70025). Phorbol 12-myristate 13-acetate, PMA (16561-29-8), PKC inhibitor, Gö6983, (133053-19-7), Cyclosporin A, CsA, (C3362), Ionomycin, (407952) were purchased from Sigma-Aldrich. Monoclonal anti-CD3 (catalog no. 555336) and anti-CD28 (catalog no. 555725) antibodies were from BD Biosciences. RPMI media without glutamine (Invitrogen, 21870-076), OptiMEM (Gibco, 31985-062), Pen Strep Glutamine (Gibco- 10378-016). Fetal Bovine Serum (Quality Biologicals, Cat 110-001-101HI).

\section{Plasmid Construction}

The plasmids pET-28b-Hoc and pET-28b-Cell Penetrating Peptide (CPP)-T-Hoc were constructed as previously described (Sathaliyawala et al., 2010; Tao et al., 2013). pET-28b-CD4 DARPin (55.2) was constructed by fusing 6X-Histidine tag to the N-terminus of CD4 DARPin, 55.2 sequence from EMBL Nucleotide Sequence Database. The sequence was synthesized from Invitrogen and amplified using following primers FW1 5' ATATACCATGGGCAGCAGCCATCATCATCATC $\quad 3, \quad$ and 1 5'TtAGgCtCGAGATtAagCTTTTGCAGGATTTCAGCCAG 3' having NcoI and XhoI cloning sites. The resulting fragment was purified by agarose gel electrophoresis, digested with appropriate restriction enzymes, and ligated with the gel-purified pET-28b vector DNA digested with the same restriction enzymes. For constructing pET28b-CD4DARPin-LYKYSD-Hoc and CD4DARPin-(GGGS) $)_{2}$ GGSA-Hoc, two rounds of PCR were done. First round for the amplification of CD4 DARPin from pET-28b-CD4 DARPin_55.2 (FW1\& BW2:) and Hoc from the pET-28b-Hoc clone (FW2: \& BW3:). Full-length CD4 DARPin-Hoc was acquired by the second round of PCR using the FW1 and BW3 primers and then digested with NcoI and XhoI. The digested fragment was subcloned into the $\mathrm{pET}-28 \mathrm{~b}$ vector. Plasmids pAAV-GFP and pAAV- 
mCherry were purchased from Cell Biolabs. All the constructed plasmids were sequenced to confirm correct fragment insertion (Retrogen, CA).

\section{CD4 DARPin-Hoc Protein expression and purification}

The recombinant proteins expressed in E. coli BL21 (DE3) RIPL cells were purified according to previously described protocols (Zhang et al., 2011). Briefly, The BL21 (DE3) RIPL cells harboring the recombinant plasmids were induced with $1 \mathrm{mM}$ IPTG for $2 \mathrm{~h}$ at $25{ }^{\circ} \mathrm{C}$. The cells were harvested by centrifugation $\left(6,000 \times \mathrm{g}\right.$ for $15 \mathrm{~min}$ at $\left.4{ }^{\circ} \mathrm{C}\right)$ and resuspended in $40 \mathrm{~mL}$ of HisTrap binding buffer (50 mM Tris $\cdot \mathrm{HCl}, \mathrm{pH} 8.0 / 20 \mathrm{mM}$ imidazole $/ 300 \mathrm{mM} \mathrm{NaCl})$. The cells were lysed using French-press (Aminco) and the soluble fraction containing the His-tagged fusion protein was isolated by centrifugation at $34,000 \times \mathrm{g}$ for 35 minutes. The supernatant was loaded onto a HisTrap column (GE Healthcare) and washed with $50 \mathrm{mM}$ imidazole containing buffer, and the protein was eluted with $20-500 \mathrm{mM}$ linear imidazole gradient. The peak fractions were collected and purified by size exclusion chromatography using Hi-Load 16/60 Superdex-200 (prep-grade) gel filtration column (GE Healthcare) in a buffer containing $25 \mathrm{mM}$ Tris $\cdot \mathrm{HCl}(\mathrm{pH}$ 8.0) and $100 \mathrm{mM} \mathrm{NaCl}$. The peak fractions were collected, flash frozen in Liquid $\mathrm{N} 2$ and stored at $-80^{\circ} \mathrm{C}$.

\section{Enzyme-linked immunosorbent assay}

ELISA plates (Evergreen Scientific, CA) were coated with $0.1 \mu \mathrm{g}$ of protein per well in coating buffer $[0.05 \mathrm{M}$ sodium carbonate-sodium bicarbonate $(\mathrm{pH} 9.6)]$ overnight at $4^{\circ} \mathrm{C}$. After washing three times with PBS-T buffer, the plates were blocked with PBS-3\% BSA buffer for 1 hour at $37^{\circ} \mathrm{C}$. The recombinant soluble CD4 (NIH AIDS Reagent program, 4615) was added and incubated for 1 hour at room temperature. The bound fraction was detected by anti-CD4 Antibody Sim.2 (NIH AIDS Reagent program, 723) by ELISA using soluble CD4 at a dilution of 1:1000. 


\section{Cell Binding assay}

Assays are performed as described previously (Chand et al., 2017). Briefly, purified CD4 DARPin variants and gp140 proteins were coated onto 96-well black, clear-bottom plate (Greiner), 15 pmoles per well, for 1 hour. The wells were subsequently washed 3 times with blocking buffer (1mM MnCl2, $0.1 \mathrm{mM} \mathrm{CaCl} 2,10 \mathrm{mM}$ HEPES, $150 \mathrm{mM} \mathrm{NaCl}$, and 10\% FBS) and then incubated with the blocking buffer for 1 hour. Wells were then washed 3 times with wash buffer (1mM MnCl2, 0.1mM CaCl2, 10mM HEPES, 150mM NaCl, and 1\% FBS). A3.01 (CD4+), A2.01 (CD4), Tzmbl and J-Lat 10.6 full length cells (50 $\mu \mathrm{l} /$ well of $4 \times 10^{6}$ cells per ml) were added in cell dilution buffer (wash buffer containing 5\% FBS) and allowed to bind for 1 hour. Wells were then washed 5 times with wash buffer and the remaining bound cells were detected with the CellTiterGlo kit (Promega) as per the manufacturer's instructions.

\section{In vitro DNA packaging and protein display of the T4 head}

For in vitro DNA packaging assays, each $20 \mu \mathrm{l}$ of reaction mixture contained purified T4 heads $\left(\sim 2 \times 10^{10}\right.$ particles), purified full-length gp17 $(\sim 3 \mu \mathrm{M})$, and linearized DNA in packaging buffer $[30 \mathrm{mM}$ tris- $\mathrm{HCl}(\mathrm{pH} 7.5), 100 \mathrm{mM} \mathrm{NaCl}, 3 \mathrm{mM} \mathrm{MgCl}$, and $1 \mathrm{mM}$ adenosine 5'triphosphate (ATP)]. The mixture was incubated at $37^{\circ} \mathrm{C}$ for $30 \mathrm{~min}$, followed by benzonase nuclease addition and incubation at $37^{\circ} \mathrm{C}$ for $20 \mathrm{~min}$ to remove excess unpackaged DNA. The encapsidated nuclease-resistant DNA was released by treatment with 50 mM EDTA, proteinase K $\left(0.5 \mathrm{~g} / \mu 1\right.$; Thermo Fisher Scientific, MA), and $0.2 \% \mathrm{SDS}$ for $30 \mathrm{~min}$ at $65^{\circ} \mathrm{C}$. The packaged DNA was analyzed by $1 \%(\mathrm{w} / \mathrm{v})$ agarose gel electrophoresis followed by staining with ethidium bromide, and the amount of packaged DNA was quantified using Quantity One software (BioRad, CA). The packaging efficiency was defined as the number of DNA molecules packaged per T4. In vitro protein display on the T4 head was assessed by the co-sedimentation described 
previously. Briefly, after encapsidating linearized DNA as described above, T4 heads were incubated with Soc- and/or Hoc-fused proteins at $4{ }^{\circ} \mathrm{C}$ for $45 \mathrm{~min}$. The mixtures were sedimented by centrifugation at 30,000g for $45 \mathrm{~min}$, and unbound proteins in the supernatants were removed. After washing twice with PBS, the pellets were incubated at $4{ }^{\circ} \mathrm{C}$ overnight and then resuspended in PBS for SDS-polyacrylamide gel electrophoresis (SDS-PAGE) analysis or Opti-MEM for transduction. After Coomassie Blue R-250 (Bio-Rad, CA) staining and destaining, the protein bands on SDS-PAGE gels were scanned and quantified by laser densitometry (Personal Densitometer SI; GE Healthcare, IL). The densities of the Hoc, Soc, and gp23 bands were determined for each lane separately, and the copy numbers of bound Hoc or Soc fusion molecules per capsid were calculated using gp23 as the internal control (930 copies per capsid).

\section{Cell transduction and the detection of gene delivery}

CD4+HEK293T cells were seeded in 24-well plates at $2.0 \times 10^{5}$ cells per well in complete DMEM. After 24 hours, the cells were incubated with the T4, CD4DARPin-T4 vectors at different multiplicities of infection in antibiotic-free Opti-MEM for 6 hours. Thereafter, Opti-MEM was removed and replaced with complete DMEM. The cells were further incubated at $37^{\circ} \mathrm{C}$ for an additional 48 hours. GFP transgene expression was observed by fluorescence microscopy (Carl Zeiss, Germany) at 48 hours after transduction. To analyze luciferase gene delivery into cells by T4 or CD4DARPin T4, we measured luciferase activity with the Luciferase Assay System (Promega, WI) according to the manufacturer's instructions. Briefly, the growth medium was removed, and cells were rinsed with PBS buffer. After removing the wash buffer, $150 \mu$ of passive lysis buffer was added to each well, followed by gentle shaking at RT for 20 mins. $20 \mu$ of the cell lysate was then transferred to a 96-well white opaque plate and mixed with $80 \mu 1$ of Luciferase 
Assay Reagent, and the luminescence signal was recorded using the GloMax- Multi Detection System (Promega, WI).

\section{Cytokine secretion analysis}

Fresh PBMCs $\left(5 \times 10^{5}\right.$ cells per well) were left unstimulated or stimulated withT4-NPs, CD4DARP-T4-NPs, and anti-CD3 \& anti-CD28 Abs for 48 hours at $37{ }^{\circ} \mathrm{C}$. Medium-treated PBMCs served as a negative control. After stimulation, cell-free supernatant was collected and analyzed by Bio-Plex ProTM Human Cytokine Th1/Th2 Assay according to the manufacturer's instructions (Bio-Rad). All tests were performed in duplicates, and mean values were calculated.

\section{Resting CD4+ $\mathbf{T}$ cell subset isolation}

Primary human CD4+ T cells were isolated from PBMCs using a Human resting CD4+ T Cells Isolation Kit I according to the manufacturer instructions (STEMCELL Technologies). Briefly, PMBCs from liquid nitrogen were equilibrated at $-800 \mathrm{C}$ for 12 hours, quickly thawed, and resuspended at $5 \times 10^{7}$ cells $/ \mathrm{mL}$ in PBS containing $2 \%$ FBS. To the sample, EasySepTM Human Resting CD4+ T Cell Isolation Cocktail, CD25 Depletion Cocktail, and Rapid Spheres were added, followed by incubation for 5 mins at room temperature. After incubation, the sample was top up to $2.5 \mathrm{ml}$ with buffer containing PBS with $2 \%$ FBS and 1mM EDTA, PBS should be without $\mathrm{Ca} 2+$ and $\mathrm{Mg} 2+$. The tube was placed into the magnet for 5 mins at $\mathrm{RT}$ and poured on in continuous motion into a new tube to get the enriched cell suspension. Purified CD4+ T cells were $>97 \%$ pure as assessed by fluorescence-activated cell sorting.

\section{Measurement of the reactivation of latent HIV-1 in the latent cells}

$2.5 \times 10^{5} \mathrm{~J}$-Lat 10.6 full-length cells were resuspended in OPTIMEM media in flat bottom 24 well plate. The cells were then treated with the indicated concentrations and the number of activators or phage T4-NPs, respectively. After 6 hours, FBS was added to make up the final 
concentration to $10 \% .2 .5 \mu \mathrm{g} / \mathrm{ml}$ anti-CD3 and $1 \mu \mathrm{g} / \mathrm{ml}$ anti-CD28 monoclonal antibodies were used as positive controls. After $40-48$ hours at $37^{\circ} \mathrm{C}$, reactivation of latent $\mathrm{HIV}-1$ was determined by quantifying the percentage of GFP+ cells using the FACS ARIA and analyzed using FlowJo (BD Biosciences) software. The percentage of GFP+ cells was calculated.

\section{HIV-specific mRNA measurements}

RNA was isolated using Direct-Zol RNA MiniPrep Plus (Zymo Research, Irvine, CA) and cDNA was synthesized by using iScript cDNA Synthesis Kit (Bio-Rad Laboratories, Hercules, CA). qRT-PCR was performed on Applied Biosystems StepOneTM Real-Time PCR System using iTaqTM Universal SYBR ${ }^{\circledR}$ green supermix (Bio-Rad Laboratories, Hercules, CA) with HIV-1 mRNA primer set: 5'-GTGTGCCCGTCTGTTGTGTGA-3'， primer $\quad$ 2: 5'GCCACTGCTAGAGAT TTTCCA-3' for and GAPDH primer set: 5'AAGGTGAAGGTCGGAGTCAAC-3' and 5'-GGGGTCATTGATGGCAACAATA-3'. The following cycling conditions were used for all qRT-PCR reactions: $95{ }^{\circ} \mathrm{C}$ for $10 \mathrm{~min}, 40$ cycles at $95^{\circ} \mathrm{C}$ for $15 \mathrm{sec}$ and $60^{\circ} \mathrm{C}$ for $1 \mathrm{~min}$. Relative fold changes were calculated after normalization with GAPDH as a reference gene.

\section{p24 ELISA}

Cell culture supernatant fluids were assayed for the Gag-derived p24 HIV protein using p24 ELISA kits (Zaptometrix) by following the manufacturer's instructions. In principle, plate wells are pre-coated with anti-p24 gag (HIV-1) antibody. Virus particles in the culture supernatants upon lysis release p24 gag protein that binds to the immobilized antibody in the well. The captured p24 protein is then detected by a biotin conjugated anti-HIV-1 antibody. Streptavidin-Peroxidase binds to biotin and the amount of HIV-1 p24 antigen in the supernatant is finally quantified by colorimetry. 


\section{HIV RNA isolation from cell supernatants}

CD4+ T cells isolated from ART suppressed patients were left untreated or treated with T4-NPs, CD4DARP-T4-NPs, anti-CD3 and anti-CD28 mAbs, and PMA. The HIV RNA from the cell released free virus in the supernatant was extracted every 3 days for up to 9 days. The cryopreserved cell supernatants were thawed quickly at $37^{\circ} \mathrm{C}, 500 \mu \mathrm{l}$ of the supernatant was diluted with Tris-buffered saline (TBS), and centrifuged at $21,100 \mathrm{xg}$ for 1 hour at $10^{\circ} \mathrm{C}$ to pellet the cell-free virus. The supernatant was then removed, and the pellet was resuspended in $100 \mathrm{ml}$ of 3M guanidinium hydrochloride $(\mathrm{GuHCl})$ containing $50 \mathrm{mM}$ Tris- $\mathrm{HCl} \mathrm{pH} 7.6,1 \mathrm{mM}$ calcium chloride and $100 \mathrm{mg}$ proteinase $\mathrm{K}$ and incubated at $42^{\circ} \mathrm{C}$ for 1 hour. Next, $400 \mathrm{ml}$ of $6 \mathrm{M}$ guanidinium thiocyanate (GuSCN) containing $50 \mathrm{mM}$ Tris $\mathrm{HCl} \mathrm{pH}$ 7.6, $1 \mathrm{mM}$ EDTA, and 600 $\mathrm{mg} / \mathrm{mL}$ glycogen was added and incubated again at $42^{\circ} \mathrm{C}$ for 10 minutes. After the second incubation, $500 \mathrm{ml}$ of $100 \%$ isopropanol at room temperature was added to the guanidinium mixture, which was then invert mixed for 20 times and centrifuged at $21,000 \mathrm{xg}$ for 15 minutes at room temperature to pellet the RNA. Then, the supernatant was removed, and the pellet was washed with $70 \%$ ethanol. RNA pellets were air-dried for 5 mins and stored at $-80^{\circ} \mathrm{C}$ for the downstream assays.

\section{cDNA synthesis and $q R T$ PCR for patient samples}

Complementary DNA (cDNA) was synthesized from $10 \mu \mathrm{l}$ of HIV RNA transcripts or extracted CA-RNA using Random hexamers and SuperScript IV Reverse transcriptase according to manufacturer protocol (Thermo Fisher). Next, a multiplexed qPCR master mix was made with a final concentration of 1X Lightcycler 480 Probes Master Mix (Roche, Switzerland), 600 nM forward and reverse primers, and $100 \mathrm{nM}$ probe. We used a primer-probe set HIV-1 gag and RCAS gag. The copy number was determined from the standard curve generated from HIV RNA 
transcript standards that were diluted from $10 \times 10^{6}$ to 1 copy/per well and assayed in triplicates for each run.

\section{Transfections of HIV-1 Env-Soc Trimers}

HEK293F suspension cells were grown overnight to a density of $10^{6}$ cells $/ \mathrm{ml}$. Large- scale transfection in $1 \mathrm{~L}$ culture volume was done using FreeStyle MAX transfection reagent (Life Technologies). Briefly, the cells were transfected with $1 \mu \mathrm{g}$ of gp140-Soc plasmid DNA/ million cells. As these clones are cleavage-sensitive, the cells were co-transfected with the furin plasmid at a gp140-Soc: furin plasmid DNA ratio of 3:1 to ensure near 100\% cleavage. Plasmid DNAs and MAX reagent were diluted in OptiPRO SFM medium, mixed and incubated for $10 \mathrm{~min}$ at room temperature. The mixture was then added to the HEK293F suspension cells. After 12 hours, the transfected cells were supplemented with $100 \mathrm{ml}$ of fresh HyClone SFM4HEK293 medium (GE Healthcare) and sodium butyrate (Reeves et al., 2002) solution (SIGMA-ALDRICH; final concentration of $2 \mathrm{nM}$ ). On day 5, the supernatant was harvested by centrifuging the cells, and filtered using a $0.2 \mu \mathrm{m}$ filter (Corning, Inc.) for purification of the secreted protein.

\section{HIV-1 gp140-soc Protein purification}

Secreted twin strep-tagged gp140-soc proteins in the harvested and filtered supernatant (1L) were supplemented with protease inhibitor tablets (Roche Diagnostics) to prevent protein degradation and $5 \mathrm{ml}$ of BioLock biotin blocking solution (iba Life Sciences $\mathrm{GmbH}$ ) to mask the biotin present in the supernatant. Next, the supernatant was loaded onto a $1 \mathrm{ml}$ Strep-Tactin column (Qiagen) at a flow rate of $0.7 \mathrm{ml} / \mathrm{min}$ in the ÄKTA prime-plus liquid chromatography system (GE Healthcare). Non-specifically bound proteins were washed off by passing at least 20 column volumes of wash buffer ( $50 \mathrm{mM}$ Tris- $\mathrm{HCl}, \mathrm{pH} 8$, and $300 \mathrm{mM} \mathrm{NaCl})$ until the absorbance reached the baseline level. Bound gp140-soc proteins were eluted with Strep-Tactin elution buffer (5 mM 
d-Desthiobiotin, $25 \mathrm{mM}$ Tris- $\mathrm{HCl}, \mathrm{pH} 8$, and $150 \mathrm{mM} \mathrm{NaCl}$ ) at a flow rate of $1 \mathrm{ml} / \mathrm{min}$. Peak protein fractions were pooled and loaded onto the size-exclusion chromatography column using Hi-Load 16/60 Superdex-200 (prep-grade) gel filtration column (GE Healthcare) in a buffer containing $25 \mathrm{mM}$ Tris $\cdot \mathrm{HCl}(\mathrm{pH} 8.0)$ and $100 \mathrm{mM} \mathrm{NaCl}$. All the eluted fractions were collected and analyzed on BLUE Native PAGE and reducing/non-reducing SDS-PAGE for biochemical analysis.

\section{Strep-Tactin ELISAs}

SEC purified HIV-1 Env-Soc trimers were tested for antigenicity using ELISA involving Microplates pre-coated with Strep-Tactin (IBA Life Sciences GmbH). Microplates were coated with $1 \mu \mathrm{g} / \mathrm{ml} \mathrm{SEC-purified} \mathrm{gp140-Soc}$ trimers in a volume of $100 \mu \mathrm{l} /$ well of coating buffer $(25$ $\mathrm{mM}$ Tris- $\mathrm{HCl}, \mathrm{pH} 7.6,2 \mathrm{mM}$ EDTA, and $140 \mathrm{mM} \mathrm{NaCl}$ ) for 2 hours at room temperature. Followed by washing with PBST $(0.05 \%$ Tween 20 in PBS) the trimers were incubated with serially diluted PGT145 and 8ANC195 antibodies for 1 hour at $37^{\circ} \mathrm{C}$. The unbound $\mathrm{Ab}$ was washed off and the bound was detected by HRP-conjugated rabbit anti-human Ab (Santa Cruz Biotechnology). Peroxidase substrate (TMB microwell peroxidase substrate system, KPL) was added and reaction was terminated by BlueSTOP solution (KPL) and OD650 was measured (Molecular Devices). 


\section{REFERENCES}

Ananthaswamy, N., Fang, Q., AlSalmi, W., Jain, S., Chen, Z., Klose, T., Sun, Y., Liu, Y., Mahalingam, M., Chand, S., et al. (2019). A sequestered fusion peptide in the structure of an HIV-1 transmitted founder envelope trimer. Nat Commun 10, 873.

Archin, N.M., Sung, J.M., Garrido, C., Soriano-Sarabia, N., and Margolis, D.M. (2014). Eradicating HIV-1 infection: seeking to clear a persistent pathogen. Nat Rev Microbiol 12, 750-764.

Auclair, S., Liu, F., Niu, Q., Hou, W., Churchyard, G., Morgan, C., Frahm, N., Nitayaphan, S., Pitisuthithum, P., Rerks-Ngarm, S., et al. (2018). Distinct susceptibility of HIV vaccine vectorinduced CD4 T cells to HIV infection. PLoS Pathog 14, e1006888.

Barr, J.J., Auro, R., Furlan, M., Whiteson, K.L., Erb, M.L., Pogliano, J., Stotland, A., Wolkowicz, R., Cutting, A.S., Doran, K.S., et al. (2013). Bacteriophage adhering to mucus provide a non-hostderived immunity. Proc Natl Acad Sci U S A 110, 10771-10776.

Black, L.W., and Rao, V.B. (2012). Structure, assembly, and DNA packaging of the bacteriophage T4 head. Adv Virus Res 82, 119-153.

Chand, S., Messina, E.L., AlSalmi, W., Ananthaswamy, N., Gao, G., Uritskiy, G., Padilla-Sanchez, V., Mahalingam, M., Peachman, K.K., Robb, M.L., et al. (2017). Glycosylation and oligomeric state of envelope protein might influence HIV-1 virion capture by alpha4beta7 integrin. Virology 508, 199-212.

Chen, Z., Sun, L., Zhang, Z., Fokine, A., Padilla-Sanchez, V., Hanein, D., Jiang, W., Rossmann, M.G., and Rao, V.B. (2017). Cryo-EM structure of the bacteriophage T4 isometric head at 3.3-A resolution and its relevance to the assembly of icosahedral viruses. Proc Natl Acad Sci U S A 114, E8184-E8193.

Chun, T.W., Davey, R.T., Jr., Engel, D., Lane, H.C., and Fauci, A.S. (1999). Re-emergence of HIV after stopping therapy. Nature 401, 874-875.

Churchill, M.J., Deeks, S.G., Margolis, D.M., Siliciano, R.F., and Swanstrom, R. (2016). HIV

reservoirs: what, where and how to target them. Nat Rev Microbiol 14, 55-60.

Duerkop, B.A., and Hooper, L.V. (2013). Resident viruses and their interactions with the immune system. Nat Immunol 14, 654-659.

Fokine, A., Chipman, P.R., Leiman, P.G., Mesyanzhinov, V.V., Rao, V.B., and Rossmann, M.G. (2004). Molecular architecture of the prolate head of bacteriophage T4. Proc Natl Acad Sci U S A 101, 6003-6008.

Fokine, A., Islam, M.Z., Zhang, Z., Bowman, V.D., Rao, V.B., and Rossmann, M.G. (2011).

Structure of the three $\mathrm{N}$-terminal immunoglobulin domains of the highly immunogenic outer capsid protein from a T4-like bacteriophage. J Virol 85, 8141-8148.

Gensollen, T., Iyer, S.S., Kasper, D.L., and Blumberg, R.S. (2016). How colonization by microbiota in early life shapes the immune system. Science 352, 539-544.

Glaria, E., and Valledor, A.F. (2020). Roles of CD38 in the Immune Response to Infection. Cells 9. Gogokhia, L., Buhrke, K., Bell, R., Hoffman, B., Brown, D.G., Hanke-Gogokhia, C., Ajami, N.J., Wong, M.C., Ghazaryan, A., Valentine, J.F., et al. (2019). Expansion of Bacteriophages Is Linked to Aggravated Intestinal Inflammation and Colitis. Cell Host Microbe 25, 285-299 e288.

Isakov, N., and Altman, A. (2002). Protein kinase C(theta) in T cell activation. Annu Rev Immunol 20, 761-794. 
Iwasaki, A., and Medzhitov, R. (2004). Toll-like receptor control of the adaptive immune responses. Nat Immunol 5, 987-995.

Jordan, A., Bisgrove, D., and Verdin, E. (2003). HIV reproducibly establishes a latent infection after acute infection of T cells in vitro. EMBO J 22, 1868-1877.

Kinet, S., Bernard, F., Mongellaz, C., Perreau, M., Goldman, F.D., and Taylor, N. (2002). gp120mediated induction of the MAPK cascade is dependent on the activation state of CD4(+) lymphocytes. Blood 100, 2546-2553.

Kinoshita, S., Su, L., Amano, M., Timmerman, L.A., Kaneshima, H., and Nolan, G.P. (1997). The T cell activation factor NF-ATc positively regulates HIV-1 replication and gene expression in T cells. Immunity 6, 235-244.

Klasse, P.J., and Moore, J.P. (2004). Is there enough gp120 in the body fluids of HIV-1-infected individuals to have biologically significant effects? Virology 323, 1-8.

Maldarelli, F., Wu, X., Su, L., Simonetti, F.R., Shao, W., Hill, S., Spindler, J., Ferris, A.L., Mellors, J.W., Kearney, M.F., et al. (2014). HIV latency. Specific HIV integration sites are linked to clonal expansion and persistence of infected cells. Science 345, 179-183.

Milletti, F. (2012). Cell-penetrating peptides: classes, origin, and current landscape. Drug Discov Today 17, 850-860.

Nguyen, S., Baker, K., Padman, B.S., Patwa, R., Dunstan, R.A., Weston, T.A., Schlosser, K., Bailey, B., Lithgow, T., Lazarou, M., et al. (2017). Bacteriophage Transcytosis Provides a Mechanism To Cross Epithelial Cell Layers. mBio 8.

Pace, M.J., Graf, E.H., Agosto, L.M., Mexas, A.M., Male, F., Brady, T., Bushman, F.D., and O'Doherty, U. (2012). Directly infected resting CD4+T cells can produce HIV Gag without spreading infection in a model of HIV latency. PLoS Pathog 8, e1002818.

Pelchen-Matthews, A., Parsons, I.J., and Marsh, M. (1993). Phorbol ester-induced downregulation of CD4 is a multistep process involving dissociation from p56lck, increased association with clathrin-coated pits, and altered endosomal sorting. J Exp Med 178, 12091222.

Perez, V.L., Rowe, T., Justement, J.S., Butera, S.T., June, C.H., and Folks, T.M. (1991). An HIV-1infected $\mathrm{T}$ cell clone defective in IL-2 production and Ca2+ mobilization after CD3 stimulation. J Immunol 147, 3145-3148.

Qin, L., Fokine, A., O'Donnell, E., Rao, V.B., and Rossmann, M.G. (2010). Structure of the small outer capsid protein, Soc: a clamp for stabilizing capsids of T4-like phages. J Mol Biol 395, 728741.

Rao, V.B., and Black, L.W. (2010). Structure and assembly of bacteriophage T4 head. Virol J 7, 356.

Ross, S.H., and Cantrell, D.A. (2018). Signaling and Function of Interleukin-2 in T Lymphocytes. Annu Rev Immunol 36, 411-433.

Salmond, G.P., and Fineran, P.C. (2015). A century of the phage: past, present and future. Nat Rev Microbiol 13, 777-786.

Sathaliyawala, T., Islam, M.Z., Li, Q., Fokine, A., Rossmann, M.G., and Rao, V.B. (2010).

Functional analysis of the highly antigenic outer capsid protein, Hoc, a virus decoration protein from T4-like bacteriophages. Mol Microbiol 77, 444-455.

Schweizer, A., Rusert, P., Berlinger, L., Ruprecht, C.R., Mann, A., Corthesy, S., Turville, S.G., Aravantinou, M., Fischer, M., Robbiani, M., et al. (2008). CD4-specific designed ankyrin repeat 
proteins are novel potent HIV entry inhibitors with unique characteristics. PLoS Pathog 4, e1000109.

Sun, S., Kondabagil, K., Draper, B., Alam, T.I., Bowman, V.D., Zhang, Z., Hegde, S., Fokine, A., Rossmann, M.G., and Rao, V.B. (2008). The structure of the phage T4 DNA packaging motor suggests a mechanism dependent on electrostatic forces. Cell 135, 1251-1262.

Tao, P., Mahalingam, M., Marasa, B.S., Zhang, Z., Chopra, A.K., and Rao, V.B. (2013). In vitro and in vivo delivery of genes and proteins using the bacteriophage T4 DNA packaging machine. Proc Natl Acad Sci U S A 110, 5846-5851.

Tao, P., Zhu, J., Mahalingam, M., Batra, H., and Rao, V.B. (2019). Bacteriophage T4 nanoparticles for vaccine delivery against infectious diseases. Adv Drug Deliv Rev 145, 57-72.

Tsai, A., Irrinki, A., Kaur, J., Cihlar, T., Kukolj, G., Sloan, D.D., and Murry, J.P. (2017). Toll-Like Receptor 7 Agonist GS-9620 Induces HIV Expression and HIV-Specific Immunity in Cells from HIV-Infected Individuals on Suppressive Antiretroviral Therapy. J Virol 91.

Vasiliver-Shamis, G., Cho, M.W., Hioe, C.E., and Dustin, M.L. (2009). Human immunodeficiency virus type 1 envelope gp120-induced partial T-cell receptor signaling creates an F-actindepleted zone in the virological synapse. J Virol 83, 11341-11355.

Walker, L.M., Huber, M., Doores, K.J., Falkowska, E., Pejchal, R., Julien, J.P., Wang, S.K., Ramos, A., Chan-Hui, P.Y., Moyle, M., et al. (2011). Broad neutralization coverage of HIV by multiple highly potent antibodies. Nature 477, 466-470.

Yanagida, M., Suzuki, Y., and Toda, T. (1984). Molecular organization of the head of bacteriophage Teven: underlying design principles. Adv Biophys 17, 97-146.

Zhang, Z., Kottadiel, V.I., Vafabakhsh, R., Dai, L., Chemla, Y.R., Ha, T., and Rao, V.B. (2011). A promiscuous DNA packaging machine from bacteriophage T4. PLoS Biol 9, e1000592. 